\title{
SMOOTH CENTER MANIFOLDS FOR RANDOM DYNAMICAL SYSTEMS
}

\author{
PENG GUO AND JUN SHEN
}

\begin{abstract}
In this paper, we will prove the existence and Hölder continuity of smooth center-unstable and centerstable manifolds for random dynamical systems based on their Lyapunov exponents. Furthermore, we obtain the existence and Hölder continuity of smooth center manifolds.
\end{abstract}

1. Introduction. The theory of invariant manifolds is a fundamental tool for describing and understanding nonlinear dynamical systems. They are widely used to investigate the qualitative behavior of flows, bifurcation characteristics and linearization, etc. The study of invariant manifolds dates back to the paper of Hadamard [16]. His method is based on graph transform. Later, Lyapunov [24] and Perron [26] used analytic method to study invariant manifolds. Since then, there has been some literature regarding invariant manifolds, including the stable, unstable, center, center-stable and center-unstable manifolds for finite- or infinite-deterministic dynamical systems, see Pliss [27], Kelley [19], Hale [17], Henry [18], Carr [6], Vanderbauwhede and van Gils [29], Chow and $\mathrm{Lu}[\mathbf{1 2}, \mathbf{1 3}]$, Bates and Jones [2], Chow, Lin and $\mathrm{Lu}[\mathbf{9}]$, Chow, Li and Wang [8], Chow, Liu and Yi [10, 11], Bates, Lu and Zeng $[\mathbf{3}, \mathbf{5}]$, etc.

Recently, there has been some work done on invariant manifolds for stochastic and random dynamical systems by Wanner [30], Arnold [1], Mohammed and Scheutzow [25], Schmalfuß [28], Duan, Lu and Schmalfuß [14], etc. Wanner's method, which is based on the Banach fixed point theorem, is essentially the Lyapunov-Perron approach.

2010 AMS Mathematics subject classification. Primary 60H10, Secondary $37 \mathrm{D} 10$.

Keywords and phrases. Random dynamical system, smoothness, center-unstable manifolds, center-stable manifolds, center manifolds.

The first author was supported by the NSFC, grant No. 11331007. The second author was supported by the NSFC, grant Nos. 11201320, 11371264 .

Received by the editors on December 23, 2014, and in revised form on February $15,2015$. 
Later, Arnold used a similar technique for studying this problem. In contrast to this method, Mohammed and Scheutzow applied the technique of semimartingales to study invariant manifolds for stochastic differential equations.

Assume that $\varphi(n, \omega, 0)=0$ for all $n \in \mathbb{Z}$ and $\omega \in \Omega$, where $(\Omega, \mathcal{F}, \mathbb{P})$ is a probability space. Then $\varphi(n, \omega, x)$ can be rewritten as

$$
\varphi(n, \omega, x)=\Phi(n, \omega) x+f(n, \omega, x),
$$

where $\Phi(n, \omega)=D \varphi(n, \omega, 0)$ and $f(n, \omega, x)$ is the higher order term. When $\Phi(n, \omega)$ is non-uniform pseudo hyperbolic, $\mathrm{Li}$ and $\mathrm{Lu}[\mathbf{2 0}]$ and Lian and $\mathrm{Lu}[\mathbf{2 1}]$ studied the existence and smoothness of stable and unstable manifolds in $\mathbb{R}^{d}$ and Banach space, respectively. When $\Phi(n, \omega)$ has zero Lyapunov exponents, Arnold [1] used the Lyapunov norm to investigate the existence and smoothness of global center manifolds. In this paper, we will use a cut-off procedure and the Lyapunov-Perron technique to prove existence and the Hölder continuity of local smooth center-unstable, center-stable and center manifolds for a nonlinear random dynamical systems $\varphi(n, \omega, x)$ in $\mathbb{R}^{d}$ and give the temperedfrom-above estimations on the derivatives of all orders. The basic ideas of our approach are to convert non-uniform exponential trichotomy into non-uniform exponential dichotomy and then use the results of center-unstable and center-stable manifolds to derive the results of center manifolds. Moreover, although the coefficient in non-uniform exponential dichotomy is a tempered random variable with the growth rate of sub-exponent, we consider a proper spectral gap and a smaller neighborhood tempered from below to ensure that existence and the Hölder continuity of smooth local invariant manifolds above without the nonlinear term having a sufficiently small Lipschitz constant.

The paper is organized as follows. In Section 2, we recall some basic concepts of random dynamical systems, the multiplicative ergodic theorem and relevant results, and then we use the cut-off function to modify the high order. In Section 3, we will successively investigate the existence, smoothness and Hölder continuity of the center-unstable, center-stable and center manifolds.

2. Random dynamical systems. In this section, we first introduce some basic concepts of random dynamical systems, the multiplicative ergodic theorem and relevant results. Then, we make some 
essential assumptions. In the end, we use the standard cut-off function to modify the nonlinear term.

Let $\mathbb{T}=\mathbb{R}$ or $\mathbb{Z}$. We denote the Borel $\sigma$-algebra on $\mathbb{R}^{d}$ and $\mathbb{T}$ by $\mathcal{B}\left(\mathbb{R}^{d}\right)$ and $\mathcal{B}(\mathbb{T})$, respectively.

Definition 2.1. $\left(\Omega, \mathcal{F}, \mathbb{P},\left(\theta^{t}\right)_{t \in \mathbb{T}}\right)$ is called a metric dynamical system if:

(i) the mapping $\theta: \mathbb{T} \times \Omega \rightarrow \Omega$ is $(\mathcal{B}(\mathbb{T}) \otimes \mathcal{F}, \mathcal{F})$-measurable;

(ii) $\theta^{0}=\operatorname{id}_{\Omega}$, the identity on $\Omega, \theta^{t+s}=\theta^{t} \circ \theta^{s}$ for all $t, s \in \mathbb{T}$;

(iii) $\theta^{t} \mathbb{P}=\mathbb{P}$ for all $t \in \mathbb{T}$.

Definition 2.2. A mapping

$$
\varphi: \mathbb{T} \times \Omega \times \mathbb{R}^{d} \longrightarrow \mathbb{R}^{d}, \quad(t, \omega, x) \mapsto \varphi(t, \omega, x)
$$

is called a random dynamical system over a metric dynamical system $\left(\Omega, \mathcal{F}, \mathbb{P},\left(\theta^{t}\right)_{t \in \mathbb{T}}\right)$ if:

(i) $\varphi$ is $\left(\mathcal{B}(\mathbb{T}) \otimes \mathcal{F} \otimes \mathcal{B}\left(\mathbb{R}^{d}\right), \mathcal{B}\left(\mathbb{R}^{d}\right)\right)$-measurable;

(ii) the mapping $\varphi(t, \omega):=\varphi(t, \omega, \cdot): \mathbb{R}^{d} \rightarrow \mathbb{R}^{d}$ forms a cocycle over $\theta^{t}$ :

$$
\varphi(0, \omega)=\text { id for all } \omega \in \Omega,
$$

$\varphi(s+t, \omega)=\varphi\left(t, \theta^{s} \omega\right) \circ \varphi(s, \omega) \quad$ for all $s, t \in \mathbb{T}$ and for all $\omega \in \Omega$.

If $\varphi$ is a random dynamical system and, for every $(t, \omega) \in \mathbb{T} \times \Omega$, the mapping

$$
\varphi(t, \omega): \mathbb{R}^{d} \longrightarrow \mathbb{R}^{d}, \quad x \mapsto \varphi(t, \omega) x
$$

is $C^{k}$ and $\varphi$ is called a $C^{k}$ smooth random dynamical system.

In this paper, we consider time-discrete $C^{N}, N \geq 2$ random dynamical systems. For every $n \in \mathbb{Z}$ and $\omega \in \Omega$ we suppose that $\varphi(n, \omega, 0)=0$. Then we rewrite $\varphi(n, \omega, x)$ as

$$
\varphi(n, \omega, x)=\Phi(n, \omega) x+f(n, \omega, x),
$$

where $D_{x} \varphi(n, \omega, 0):=\Phi(n, \omega) \in \operatorname{Gl}(d ; \mathbb{R})$ and the nonlinear term $f(n, \omega, \cdot)$ and its derivative vanish at $x=0$. By the cocycle property of $\varphi, \Phi(n, \omega)$ is a linear cocycle with two-sided time over $\left(\Omega, \mathcal{F}, \mathbb{P},\left(\theta^{n}\right)_{n \in \mathbb{Z}}\right)$. 
Let $A(\omega):=\Phi(1, \omega)$ and $F(\omega, x):=f(1, \omega, x)$. Then the time-one $\operatorname{map} \varphi(1, \omega) x$ can be written as

$$
\varphi(1, \omega) x=A(\omega) x+F(\omega, x)
$$

where $F(\omega, 0)=0$ and $D_{x} F(\omega, 0)=0$. In addition, $\Phi(n, \omega)$ is generated by $A(\omega)$ :

$$
\Phi(n, \omega)= \begin{cases}A\left(\theta^{n-1} \omega\right) \cdots A(\omega), & n>0 \\ I, & n=0 \\ A^{-1}\left(\theta^{n} \omega\right) \cdots A^{-1}\left(\theta^{-1} \omega\right), & n<0\end{cases}
$$

Let $\left\{x_{n}\right\}_{n \in \mathbb{Z}}$ be an orbit of $\varphi(n, \omega, x)$ with initial value $x_{0}$, i.e., $x_{n}:=$ $\varphi\left(n, \omega, x_{0}\right)$. Then, $\left\{x_{n}\right\}_{n \in \mathbb{Z}}$ satisfies the equation

$$
x_{n+1}=A\left(\theta^{n} \omega\right) x_{n}+F\left(\theta^{n} \omega, x_{n}\right) .
$$

Next we give a discrete variation of constants formula.

Lemma 2.3 (Discrete variations of constant formula). Assume that $\left\{x_{n}\right\}_{n \in \mathbb{Z}^{+}}$is a positive orbit of $\varphi(n, \omega, x)$. Then $\left\{x_{n}\right\}_{n \in \mathbb{Z}^{+}}$satisfies $(2.2)$

$$
x_{n}=\Phi(n, \omega) x_{0}+\sum_{i=0}^{n-1} \Phi\left(n-1-i, \theta^{i+1} \omega\right) F\left(\theta^{i} \omega, x_{i}\right) \quad \text { for all } n \geq 1 \text {. }
$$

Let $\left\{x_{n}\right\}_{n \in \mathbb{Z}^{-}}$be a negative orbit of $\varphi(n, \omega, x)$. For every $k<n$, $\left\{x_{n}\right\}_{n \in \mathbb{Z}^{-}}$satisfies

$$
x_{n}=\Phi\left(n-k, \theta^{k} \omega\right) x_{k}+\sum_{i=k}^{n-1} \Phi\left(n-1-i, \theta^{i+1} \omega\right) F\left(\theta^{i} \omega, x_{i}\right)
$$

This lemma is from [20]. The details are omitted here for brevity.

The following concept plays an important role in the study of random dynamical systems. 


\section{Definition 2.4.}

(i) A random variable $R:(\Omega, \mathcal{F}) \rightarrow\left(\mathbb{R}^{+} \backslash\{0\}, \mathcal{B}\left(\mathbb{R}^{+} \backslash\{0\}\right)\right)$ is called tempered with respect to a metric dynamical system $\theta^{n}$ if

$$
\lim _{n \rightarrow \pm \infty} \frac{1}{n} \log R\left(\theta^{n} \omega\right)=0 \mathbb{P} \text {-almost surely. }
$$

(ii) A random variable $R:(\Omega, \mathcal{F}) \rightarrow\left(\mathbb{R}^{+}, \mathcal{B}\left(\mathbb{R}^{+}\right)\right)$is called tempered from above if

$$
\lim _{n \rightarrow \pm \infty} \frac{1}{n} \log ^{+} R\left(\theta^{n} \omega\right)=0 \mathbb{P} \text {-almost surely. }
$$

(iii) A random variable $R:(\Omega, \mathcal{F}) \rightarrow((0,+\infty], \mathcal{B}(0,+\infty])$ is called tempered from below if $1 / R$ is tempered from above.

Moreover, we recall that a multifunction $W=\{W(\omega)\}_{\omega \in \Omega}$ of nonempty closed sets $W(\omega), \omega \in \Omega$, contained in $\mathbb{R}^{d}$ is called a random set if

$$
\omega \longmapsto \inf _{y \in W(\omega)}|x-y|
$$

is a random variable for every $x \in \mathbb{R}^{d}$.

Definition 2.5. A random set $W(\omega)$ is called an invariant set for a random dynamical system $\varphi(n, \omega, x)$ if

$$
\varphi(n, \omega, W(\omega))=W\left(\theta^{n} \omega\right) \quad \text { for all } n \in \mathbb{Z} .
$$

The following theorem is the multiplicative ergodic theorem $[\mathbf{1}$, pages 134,153$]$.

Theorem 2.6 (Multiplicative ergodic theorem). Let $\Phi$ be a linear random dynamical system over the metric dynamical system $(\Omega, \mathcal{F}, \mathbb{P}$, $\left.\left(\theta^{n}\right)_{n \in \mathbb{Z}}\right)$. Assume that

$$
\log ^{+}\|A(\cdot)\| \in L^{1}(\Omega, \mathcal{F}, \mathbb{P}), \quad \log ^{+}\left\|A^{-1}(\cdot)\right\| \in L^{1}(\Omega, \mathcal{F}, \mathbb{P}) .
$$

Then there exists an invariant subset $\widetilde{\Omega} \subset \Omega$ of full measure such that for each $\omega \in \widetilde{\Omega}$ the following hold:

(i) the $\lim _{n \rightarrow+\infty}\left(\Phi(n, \omega)^{*} \Phi(n, \omega)\right)^{1 / 2 n}=: \Psi(\omega)>0$ exists. 
(ii) Let $e^{\lambda_{p(\omega)}(\omega)}<\cdots<e^{\lambda_{1}(\omega)}$ be the different eigenvalues of $\Psi(\omega)$, and let $U_{p(\omega)}(\omega), \ldots, U_{1}(\omega)$ be the corresponding eigenspaces with multiplicities $d_{i}(\omega):=\operatorname{dim} U_{i}(\omega)$. Then:

$$
\begin{aligned}
p(\theta \omega) & =p(\omega), \\
\lambda_{i}(\theta \omega) & =\lambda_{i}(\omega) \quad \text { for all } i \in\{1, \ldots, p(\omega)\}, \\
d_{i}(\theta \omega) & =d_{i}(\omega) \quad \text { for all } i \in\{1, \ldots, p(\omega)\}, \\
\mathbb{R}^{d} & =U_{1}(\omega) \oplus \cdots \oplus U_{p(\omega)}(\omega) .
\end{aligned}
$$

(iii) If $\left(\Omega, \mathcal{F}, \mathbb{P},\left(\theta^{n}\right)_{n \in \mathbb{Z}}\right)$ is ergodic, the functions $p(\omega), \lambda_{i}(\omega)$ and $d_{i}(\omega)$ are constant on $\widetilde{\Omega}$.

(iv) For each $\omega \in \widetilde{\Omega}$, there exists a splitting

$$
\mathbb{R}^{d}=E_{1}(\omega) \oplus \cdots \oplus E_{p(\omega)}(\omega)
$$

of $\mathbb{R}^{d}$ into random subspaces $E_{i}(\omega)$ with dimension $d_{i}(\omega)$. Moreover, if $P^{i}(\omega): \mathbb{R} \rightarrow E_{i}(\omega)$ denotes the corresponding projection onto $E_{i}(\omega)$, then

$$
A(\omega) P^{i}(\omega)=P^{i}(\theta \omega) A(\omega),
$$

equivalently,

$$
A(\omega) E_{i}(\omega)=E_{i}(\theta \omega)
$$

(v) We have

$$
\lim _{n \rightarrow \pm \infty} \frac{1}{n} \log |\Phi(n, \omega) x|=\lambda_{i}(\omega) \Longleftrightarrow x \in E_{i}(\omega) \backslash\{0\} .
$$

(vi) The functions $\omega \mapsto p(\omega) \in\{1, \ldots, d\}, \omega \mapsto \lambda_{i}(\omega) \in \mathbb{R}, \omega \mapsto$ $d_{i}(\omega) \in\{1, \ldots, d\}, \omega \mapsto E_{i}(\omega)$ and $\omega \mapsto P^{i}(\omega)$ are measurable.

Here $\lambda_{i}(\omega)$ and $E_{i}(\omega)$ are so-called Lyapunov exponents and Oseledet spaces, respectively.

In the remainder of this paper, we denote $\widetilde{\Omega}$ by $\Omega$ and assume that all statements are true for $\omega \in \Omega$.

From now on, we always assume the following.

Hypothesis 2.7. $\Phi(n, \omega)=D_{x} \varphi(n, \omega, 0)$ satisfies the multiplicative ergodic theorem. 
We divide the Lyapunov exponents into three groups based on their signs. Let

$$
\begin{aligned}
\sigma_{u}(\omega) & :=\left\{\lambda_{i}(\omega)>0\right\}, \\
\sigma_{s}(\omega) & :=\left\{\lambda_{i}(\omega)<0\right\}, \\
\sigma_{c}(\omega) & :=\left\{\lambda_{i}(\omega)=0\right\},
\end{aligned}
$$

and denote

$$
\begin{aligned}
& E^{u}(\omega):=\bigoplus_{\lambda_{i}(\omega) \in \sigma_{u}(\omega)} E_{i}(\omega), \\
& E^{s}(\omega):=\bigoplus_{\lambda_{i}(\omega) \in \sigma_{s}(\omega)} E_{i}(\omega), \\
& E^{c}(\omega):=\bigoplus_{\lambda_{i}(\omega) \in \sigma_{c}(\omega)} E_{i}(\omega),
\end{aligned}
$$

with corresponding projections

$$
\begin{aligned}
P^{u}(\omega) & : \mathbb{R}^{d} \longmapsto E^{u}(\omega), \\
P^{s}(\omega) & : \mathbb{R}^{d} \longmapsto E^{s}(\omega), \\
P^{c}(\omega) & : \mathbb{R}^{d} \longmapsto E^{c}(\omega) .
\end{aligned}
$$

Then

$$
\mathbb{R}^{d}=E^{u}(\omega) \oplus E^{s}(\omega) \oplus E^{c}(\omega)
$$

We call $E^{u}(\omega)$ the unstable Oseledets subspace, $E^{s}(\omega)$ the stable Oseledets subspace and $E^{c}(\omega)$ the center Oseledets subspace. Let $d_{u}(\omega)$, $d_{s}(\omega)$ and $d_{c}(\omega)$ denote the dimensions of $E^{u}(\omega), E^{s}(\omega)$ and $E^{c}(\omega)$, respectively. From Theorem 2.6, $d_{u}(\omega), d_{s}(\omega)$ and $d_{c}(\omega)$ are measurable functions from $\Omega$ to $\{1, \ldots, d\}$, and $P^{s}(\omega), P^{u}(\omega)$ and $P^{c}(\omega)$ are measurable projections. Thus, $\Omega$ can be decomposed into a union of $l$ disjoint $\theta$-invariant measurable sets:

$$
\Omega=\bigcup_{i=1}^{l} \Omega_{i},
$$

where, on each $\Omega_{i}, d_{u}(\omega), d_{s}(\omega)$ and $d_{c}(\omega)$ are constant. We will build the center-unstable manifold, the center-stable manifold and the center manifold over $\Omega_{i}$. Then we may patch them together to get dimensionvarying invariant manifolds on the whole $\Omega$. In particular, when 
$\left(\Omega, \mathcal{F}, \mathbb{P},\left(\theta^{n}\right)_{n \in \mathbb{Z}}\right)$ is ergodic, $d_{u}(\omega), d_{s}(\omega)$ and $d_{c}(\omega)$ are all constant over $\Omega$.

To assure the existence of center manifolds, we need to assume that

Hypothesis 2.8. $\sigma_{c}(\omega) \neq \emptyset$, i.e., $\Phi(n, \omega)$ has zero Lyapunov exponents.

The next lemma is a consequence of the multiplicative ergodic Theorem 2.6.

Lemma 2.9. There exist $\theta$-invariant random variable $\beta(\omega)>0$, constant $\kappa$ and tempered random variable $K(\omega): \Omega \rightarrow[1,+\infty)$ such that

$$
\begin{aligned}
& \left\|\Phi(n, \omega) P^{u}(\omega)\right\| \leq K(\omega) e^{\beta(\omega) n} \quad \text { for all } n \leq 0, \\
& \left\|\Phi(n, \omega) P^{s}(\omega)\right\| \leq K(\omega) e^{-\beta(\omega) n} \quad \text { for all } n \geq 0 \text {, } \\
& \left\|\Phi(n, \omega) P^{c}(\omega)\right\| \leq K(\omega) e^{\kappa|n|} \quad \text { for all } n \in \mathbb{Z} \text {. }
\end{aligned}
$$

Proof. We choose

$$
\beta(\omega):=\min \left\{\left|\lambda_{i}(\omega)\right|: \lambda_{i}(\omega) \in \sigma_{u}(\omega) \cup \sigma_{s}(\omega), i=1, \ldots, p(\omega)\right\}-\kappa,
$$

where $\kappa$ is small such that $\beta(\omega)>0$. By [1, Corollary 4.3.5], there exists a tempered random variable $K^{\prime}(\omega)$ such that, for each $i=1, \ldots, p(\omega)$, as $x \in E_{i}(\omega)$,

$$
\frac{1}{K^{\prime}(\omega)} e^{\lambda_{i}(\omega) n-\kappa|n|}|x| \leq|\Phi(n, \omega) x| \leq K^{\prime}(\omega) e^{\lambda_{i}(\omega) n+\kappa|n|}|x| .
$$

Taking $K(\omega):=d K^{\prime}(\omega)$, we complete the proof.

As $\omega$ varies, $\beta(\omega)$ may be arbitrarily small and $K(\omega)$ may be arbitrarily large. However, along each orbit $\theta^{n} \omega, \beta(\omega)$ is a constant and $K(\omega)$ can increase only at a subexponential rate.

Although the invariant splitting of $\mathbb{R}^{d}=E_{1}(\omega) \oplus \ldots \oplus E_{p(\omega)}(\omega)$ depends on the sample point, by [1, Corollary 4.3.12], we can choose a new coordinate system so that the corresponding Oseledet spaces are deterministic. We restate the facts as follows. 
Lemma 2.10. There exists a measurable map

$$
P: \Omega \longrightarrow \operatorname{Gl}(d, \mathbb{R})
$$

such that:

(i) $\Phi(n, \omega)$ is conjugate to a block diagonal random dynamical system, i.e.,

$$
P\left(\theta^{n} \omega\right) \Phi(n, \omega) P^{-1}(\omega)=\Psi(n, \omega)=\operatorname{diag}\left(\Psi_{1}(n, \omega), \ldots, \Psi_{p(\omega)}(n, \omega)\right),
$$

where $\Psi_{i}(n, \omega)$ are cocycles of size $d_{i}(\omega)$.

(ii) The transformation $P$ preserves the Lyapunov spectrum

$$
\left\{\left(\lambda_{i}(\omega), d_{i}(\omega)\right) \mid 1 \leq i \leq p(\omega)\right\}
$$

and the corresponding Oseledet spaces

$$
\widetilde{E}_{i}(\omega)=\{0\} \times \cdots \times\{0\} \times \mathbb{R}^{d_{i}(\omega)} \times\{0\} \times \cdots \times\{0\} \subset \mathbb{R}^{d} .
$$

(iii) $\|P(\omega)\|$ and $\left\|P^{-1}(\omega)\right\|$ are tempered.

From Lemma 2.10, we know that the unstable, stable and center Oseledet subspaces for $\Psi$ are:

$$
\begin{aligned}
& \widetilde{E}^{u}(\omega):=\bigoplus_{\lambda_{i}(\omega) \in \sigma_{u}(\omega)} \widetilde{E}_{i}(\omega), \\
& \widetilde{E}^{s}(\omega):=\bigoplus_{\lambda_{i}(\omega) \in \sigma_{s}(\omega)} \widetilde{E}_{i}(\omega), \\
& \widetilde{E}^{c}(\omega):=\bigoplus_{\lambda_{i}(\omega) \in \sigma_{c}(\omega)} \widetilde{E}_{i}(\omega) .
\end{aligned}
$$

Then $\mathbb{R}^{d}$ has orthogonal decomposition:

$$
\mathbb{R}^{d}=\widetilde{E}^{u}(\omega) \oplus \widetilde{E}^{s}(\omega) \oplus \widetilde{E}^{c}(\omega) .
$$

We still denote $P^{u}(\omega)$ and $P^{s}(\omega)$ by the corresponding projections. The next lemma is a direct result of Lemmas 2.9 and 2.10.

Lemma 2.11. Assume that Hypotheses 2.7 and 2.8 hold. There exist random variables $\beta: \Omega \rightarrow(0,+\infty)$ and constant $\alpha>0$ satisfying $\alpha<$ $\beta(\omega) /\left[2(N+1)^{2}\right]$ and a tempered random variable $K(\omega): \Omega \rightarrow[1,+\infty)$ 
such that:

$$
\begin{aligned}
\left\|\Psi(n, \omega) P^{u}(\omega)\right\| & \leq K(\omega) e^{\beta(\omega) n} & & \text { for all } n \leq 0 \\
\left\|\Psi(n, \omega) P^{s}(\omega)\right\| & \leq K(\omega) e^{-\beta(\omega) n} & & \text { for all } n \geq 0 \\
\left\|\Psi(n, \omega) P^{c}(\omega)\right\| & \leq K(\omega) e^{\alpha|n|} & & \text { for all } n \in \mathbb{Z} .
\end{aligned}
$$

Denote

$$
\begin{array}{llrl}
P^{c u}(\omega) & =P^{c}(\omega)+P^{u}(\omega), & & P^{c s}(\omega)=P^{c}(\omega)+P^{s}(\omega), \\
E^{c u}(\omega)=E^{c}(\omega) \oplus E^{u}(\omega), & E^{c s}(\omega)=E^{c}(\omega) \oplus E^{s}(\omega) .
\end{array}
$$

We call $E^{c u}(\omega)$ center-unstable Oseledet subspaces and $E^{c s}(\omega)$ centerstable Oseledet subspaces. On $\Omega_{i}$, by Lemma $2.10, \mathbb{R}^{d}$ has an invariant splitting $\mathbb{R}^{d}=E^{c u} \oplus E^{s}\left(\mathbb{R}^{d}=E^{c s} \oplus E^{u}\right)$ independent of $\omega$. Surely, the projection operators $P^{c u}, P^{s}\left(P^{c s}, P^{u}\right)$ are also independent of $\omega$ on $\Omega_{i}$. For each $x \in \mathbb{R}^{d}$, we can decompose it to

$$
\begin{aligned}
& x=x^{c u}+x^{s} \text { for some } x^{c u} \in E^{c u} \text { and } x^{s} \in E^{s}, \\
& x=x^{c s}+x^{u} \text { for some } x^{c s} \in E^{c s} \text { and } x^{u} \in E^{u} .
\end{aligned}
$$

To simplify the notation, we will use $\Omega$ to denote $\Omega_{i}$. By Lemma 2.11, we obtain the next corollary.

Corollary 2.12. Assume that Hypotheses 2.7 and 2.8 hold. There exist random variables $\alpha, \beta: \Omega \rightarrow(0,+\infty)$ satisfying $\alpha(\omega)<\beta(\omega) /[2(N+$ $\left.1)^{2}\right]$ and a tempered random variable $K(\omega): \Omega \rightarrow[1,+\infty)$ such that:

$$
\begin{aligned}
& \left\|\Psi(n, \omega) P^{c u}(\omega)\right\| \leq K(\omega) e^{-\alpha(\omega) n} \quad \text { for all } n \leq 0, \\
& \left\|\Psi(n, \omega) P^{s}(\omega)\right\| \leq K(\omega) e^{-\beta(\omega) n} \quad \text { for all } n \geq 0,
\end{aligned}
$$

and

$$
\begin{aligned}
& \left\|\Psi(n, \omega) P^{u}(\omega)\right\| \leq K(\omega) e^{\beta(\omega) n} \quad \text { for all } n \leq 0, \\
& \left\|\Psi(n, \omega) P^{c s}(\omega)\right\| \leq K(\omega) e^{\alpha(\omega) n} \quad \text { for all } n \geq 0 \text {. }
\end{aligned}
$$

The random linear transformation $P(\omega)$ transforms the random dynamical system $\varphi(n, \omega, x)$ to

$$
\widetilde{\varphi}(n, \omega, x)=P\left(\theta^{n} \omega\right) \varphi\left(n, \omega, P^{-1}(\omega) x\right)=\Psi(n, \omega) x+\widetilde{f}(n, \omega, x),
$$


where $\tilde{f}(n, \omega, x)=P\left(\theta^{n} \omega\right) f\left(n, \omega, P^{-1}(\omega) x\right)$. Without loss of generality, under Hypotheses 2.7 and 2.8, we always obtain that the linear part $\Phi(n, \omega)$ of random dynamical system $\varphi(n, \omega, x)$ satisfies Lemma 2.11 and Corollary 2.12. Moreover, we fix the $\theta$-invariant random variable $\Theta(\omega): \Omega \rightarrow(0,+\infty)$, which will be determined later. By [20, Lemma 2.7 , we can choose $K(\omega)$ such that

$$
e^{-\Theta(\omega)|n|} K(\omega) \leq K\left(\theta^{n} \omega\right) \leq e^{\Theta(\omega)|n|} K(\omega) .
$$

Moreover, now we need to generalize the results in [20, Lemma 2.7] for the following case.

Lemma 2.13. Let $f: \Omega \rightarrow(0,+\infty)$ be tempered from above and $\sigma: \Omega \rightarrow(0,+\infty)$ a $\theta$-invariant random variable. Then there exists a random variable tempered from above $R(\omega)$ such that:

(i) $f(\omega) \leq R(\omega)$,

(ii) $R\left(\theta^{n} \omega\right) \leq e^{\sigma(\omega)|n|} R(\omega)$.

Proof. Since $f$ is tempered from above, there is for each $\varepsilon$ a $C_{\varepsilon}$ with

$$
f\left(\theta^{n} \omega\right) \leq C_{\varepsilon}(\omega) e^{\varepsilon|n|}
$$

Set

$$
R_{\varepsilon}(\omega):=\sup _{n \in \mathbb{Z}}\left\{1, f\left(\theta^{n} \omega\right) e^{-\varepsilon|n|}\right\}
$$

Clearly, $1 \leq R_{\varepsilon}(\omega)<\infty$. Then,

$$
f\left(\theta^{n} \omega\right) \leq R_{\varepsilon}(\omega) e^{\varepsilon|n|}
$$

It is obvious that $R_{\varepsilon}$ satisfies (i). Next, we prove that $R_{\varepsilon}(\omega)$ is tempered from above. Note that, for all $n, m \in \mathbb{Z}$,

$$
f\left(\theta^{n+m} \omega\right) \leq R_{\varepsilon}(\omega) e^{\varepsilon|m|} e^{\varepsilon|n|}
$$

and

$$
f\left(\theta^{n+m} \omega\right)=f\left(\theta^{n}\left(\theta^{m} \omega\right)\right) \leq R_{\varepsilon}\left(\theta^{m} \omega\right) e^{\varepsilon|n|} .
$$

So, for all $m \in \mathbb{Z}$, we get that

$$
R_{\varepsilon}\left(\theta^{m} \omega\right) \leq R_{\varepsilon}(\omega) e^{\varepsilon|m|},
$$


which yields

$$
\limsup _{m \rightarrow \pm \infty} \frac{1}{|m|} \log R_{\varepsilon}\left(\theta^{m} \omega\right) \leq \varepsilon .
$$

Following $R_{\varepsilon}(\omega) \geq 1$, we have

$$
\limsup _{m \rightarrow \pm \infty} \frac{1}{|m|} \log ^{+} R_{\varepsilon}\left(\theta^{m} \omega\right)=\limsup _{m \rightarrow \pm \infty} \frac{1}{|m|} \log R_{\varepsilon}\left(\theta^{m} \omega\right)=0,
$$

where the second equality is a consequence of [1, Proposition 4.1.3]. Then $R_{\varepsilon}(\omega)$ is tempered from above. For $k \in \mathbb{N}$, let

$$
\widetilde{\Omega}_{k}=\left\{\omega \in \Omega \mid \frac{1}{k} \leq \sigma(\omega)<\frac{1}{k-1}\right\} .
$$

By the above analysis, on each $\widetilde{\Omega}_{k}$, there exists a random variable tempered from above $R_{1 / k}$ such that

$$
f\left(\theta^{n} \omega\right) \leq R_{1 / k}(\omega) e^{|n| / k} \leq R_{1 / k}(\omega) e^{\sigma(\omega)|n|} .
$$

Define a random variable $R: \Omega \rightarrow(0,+\infty)$ by $R(\omega)=R_{1 / k}(\omega)$ for $\omega \in \widetilde{\Omega}_{k}$. Hence, Lemma 2.13 is established.

For the nonlinear term $f(1, \omega, x)$, we suppose that:

Hypothesis 2.14. There exists a ball

$$
U(\omega)=B\left(0, \rho_{0}(\omega)\right)=\left\{x \in \mathbb{R}^{d}|| x \mid<\rho_{0}(\omega)\right\},
$$

where $\rho_{0}: \Omega \rightarrow(0,+\infty)$ is tempered from below such that

$$
\begin{gathered}
\sup _{x \in U(\omega)}\left\|D_{x}^{k} f(1, \omega, x)\right\|_{L^{k}\left(\mathbb{R}^{d}, \mathbb{R}^{d}\right)} \leq \widetilde{B}_{k}(\omega) \\
\quad \text { for all } 0 \leq k \leq N<+\infty, \quad \omega \in \Omega,
\end{gathered}
$$

where the $\widetilde{B}_{k}$ are tempered from above and the $L^{k}\left(\mathbb{R}^{d}, \mathbb{R}^{d}\right)$ are the Banach space of all $k$-linear maps from $\mathbb{R}^{d}$ to $\mathbb{R}^{d}$ with the norm $\|\cdot\|_{L^{k}\left(\mathbb{R}^{d}, \mathbb{R}^{d}\right)} \cdot$

In addition, to investigate the Hölder continuity of smooth invariant manifolds, we also assume that: 
Hypothesis 2.15. There are tempered-from-above random $\widetilde{A}: \Omega \rightarrow$ $(0,+\infty)$ and $\theta$-invariant random variables $\tau: \Omega \rightarrow(0,1]$ such that, for each $\omega \in \Omega$,

$$
\begin{gathered}
\left\|D_{x}^{N} f(1, \omega, x)-D_{x}^{N} f(1, \omega, y)\right\|_{L^{N}\left(\mathbb{R}^{d}, \mathbb{R}^{d}\right)} \leq \widetilde{A}(\omega)|x-y|^{\tau(\omega)} \\
\text { for all } x, y \in U(\omega) .
\end{gathered}
$$

Then, we introduce the cut-off function to modify the nonlinear term $f(1, \omega, x)$. Let $\sigma(s)$ be a $C^{\infty}$ function from $(-\infty,+\infty)$ to $[0,1]$ with

$$
\begin{gathered}
\sigma(s)=1 \quad \text { for }|s| \leq 1 \\
\sigma(s)=0 \quad \text { for }|s| \geq 2, \\
\sup _{s \in \mathbb{R}}\left|\sigma^{\prime}(s)\right| \leq 2
\end{gathered}
$$

Assume that $\rho: \Omega \rightarrow(0,+\infty)$ is a random variable tempered from below such that $2 \rho(\omega) \leq \rho_{0}(\omega)$. We modify $f(1, \omega, x)$ as follows.

$$
F_{\rho}(\omega, x)= \begin{cases}\sigma(|x| / \rho(\omega)) f(1, \omega, x) & |x| \leq 2 \rho(\omega), \\ 0 & |x|>2 \rho(\omega) .\end{cases}
$$

Then $f(1, \omega, \cdot)$ is extended to the outside of $U(\omega)$. By a simple calculation, we obtain the following.

\section{Lemma 2.16.}

(i) $F_{\rho}(\omega, x)=f(1, \omega, x)$ for all $|x| \leq \rho(\omega)$;

(ii) $\left\|D_{x} F_{\rho}(\omega, x)\right\|_{L\left(\mathbb{R}^{d}, \mathbb{R}^{d}\right)} \leq 10 \widetilde{B}_{2}(\omega) \rho(\omega)$ for all $\omega \in \Omega$ and $x \in \mathbb{R}^{d}$;

(iii) $\sup _{x \in \mathbb{R}^{d}}\left\|D_{x}^{k} F_{\rho}(\omega, x)\right\|_{L^{k}\left(\mathbb{R}^{d}, \mathbb{R}^{d}\right)} \leq B_{k}(\omega)$ for all $2 \leq k \leq N$ and $\omega \in \Omega$, where each $B_{k}(\omega)$ is a random variable tempered from above.

(iv) $\left\|D_{x}^{k} F_{\rho}(\omega, x)-D_{x}^{k} F_{\rho}(\omega, y)\right\|_{L^{k}\left(\mathbb{R}^{d}, \mathbb{R}^{d}\right)} \leq A_{k}(\omega)|x-y|^{\tau(\omega)}$ for all $0 \leq k \leq N$ and $x, y \in \mathbb{R}^{d}$, where each $A_{k}(\omega)$ is random variable tempered from above.

Set

$$
L_{1}(\alpha, \beta):=\frac{e^{\beta(\omega)}}{\beta(\omega) /\left(2(N+1)^{2}\right)-\alpha(\omega)}+\frac{2}{\beta(\omega)} e^{\beta(\omega)}
$$


We choose $\rho(\omega)$ such that

$$
\rho(\omega) \leq \min \left\{\frac{L_{1}(\alpha, \beta)^{-1}}{30 K(\theta \omega) K(\omega) \widetilde{B}_{2}(\omega)}, \frac{1}{10 K(\theta \omega) K(\omega) \widetilde{B}_{2}(\omega)},\right.
$$

The choice of numbers here is not optimal but is for convenience. Let $B_{1}(\omega):=(K(\theta \omega) K(\omega))^{-1}$. The condition

$$
\left\|D_{x} F_{\rho}(\omega, x)\right\| \leq \frac{1}{2 K(\theta \omega) e^{|\beta(\omega)|}}
$$

implies that

$$
\psi(1, \omega, x):=\Phi(1, \omega) x+F_{\rho}(\omega, x)
$$

is a $C^{N}$ diffeomorphism on $\mathbb{R}^{d}$. Then $\psi(1, \omega, x)$ generates a $C^{N}$ modified random dynamical system.

Recall that $\left\{x_{n}\right\}_{n \in \mathbb{Z}}$ is an orbit of $\varphi\left(n, \omega, x_{0}\right)$ if and only if

$$
x_{n+1}=\Phi\left(1, \theta^{n} \omega\right) x_{n}+f\left(1, \theta^{n} \omega, x_{n}\right) \text { for all } n \in \mathbb{Z} .
$$

Then by Lemma 2.16 (i), we obtain that, for every sequence $x_{n} \in$ $B\left(0, \rho\left(\theta^{n} \omega\right)\right), x_{n}=\varphi\left(n, \omega, x_{0}\right)$ if and only if

$$
x_{n+1}=\Phi\left(1, \theta^{n} \omega\right) x_{n}+F_{\rho}\left(\theta^{n} \omega, x_{n}\right) \quad \text { for all } n \in \mathbb{Z} .
$$

From now on, we consider modified equation (2.5). To simplify the notation, the modified random dynamical system is still denoted by $\varphi(n, \omega, x)$.

Let $\gamma: \Omega \rightarrow \mathbb{R}^{-}$be a $\theta$-invariant random variable. We define the Banach spaces

$$
C_{\gamma(\omega)}^{+}:=\left\{\mathbf{x}=\left\{x_{n}\right\}_{n \in \mathbb{Z}^{+}}\left|x_{n} \in \mathbb{R}^{d}, \sup _{n \in \mathbb{Z}^{+}}\right| x_{n} \mid e^{\gamma(\omega) n}<+\infty\right\}
$$

with the norm

$$
\begin{gathered}
|\mathbf{x}|_{\gamma(\omega)}^{+}:=\sup _{n \in \mathbb{Z}^{+}}\left|x_{n}\right| e^{\gamma(\omega) n} \\
C_{\gamma(\omega)}^{-}:=\left\{\mathbf{x}=\left\{x_{n}\right\}_{n \in \mathbb{Z}^{-}}\left|x_{n} \in \mathbb{R}^{d}, \sup _{n \in \mathbb{Z}^{-}}\right| x_{n} \mid e^{-\gamma(\omega) n}<+\infty\right\}
\end{gathered}
$$


with the norm

$$
|\mathbf{x}|_{\gamma(\omega)}^{-}:=\sup _{n \in \mathbb{Z}^{-}}\left|x_{n}\right| e^{-\gamma(\omega) n}
$$

and

$$
C_{\gamma(\omega)}:=\left\{\mathbf{x}=\left\{x_{n}\right\}_{n \in \mathbb{Z}}\left|x_{n} \in \mathbb{R}^{d}, \sup _{n \in \mathbb{Z}}\right| x_{n} \mid e^{\gamma(\omega)|n|}<+\infty\right\}
$$

with the norm

$$
|\mathbf{x}|_{\gamma(\omega)}:=\sup _{n \in \mathbb{Z}}\left|x_{n}\right| e^{\gamma(\omega)|n|} .
$$

For the modified random dynamical system $\varphi$, we define the centerstable set as

$$
W^{c s}(\omega):=\left\{x_{0} \in \mathbb{R}^{d} \mid \mathbf{x}=\left\{x_{n}\right\}_{n \in \mathbb{Z}^{+}} \in C_{\gamma(\omega)}^{+}\right\},
$$

the center-unstable set as

$$
W^{c u}(\omega):=\left\{x_{0} \in \mathbb{R}^{d} \mid \mathbf{x}=\left\{x_{n}\right\}_{n \in \mathbb{Z}^{-}} \in C_{\gamma(\omega)}^{-}\right\}
$$

and the center set as

$$
W^{c}(\omega):=\left\{x_{0} \in \mathbb{R}^{d} \mid \mathbf{x}=\left\{x_{n}\right\}_{n \in \mathbb{Z}} \in C_{\gamma(\omega)}\right\} .
$$

Obviously, $W^{c s}(\omega), W^{c u}(\omega)$ and $W^{c}(\omega)$ are invariant for the random dynamical system $\varphi(n, \omega, x)$.

3. Center-unstable, center-stable and center manifolds. In this section, we will show the existence and smoothness of centerunstable, center-stable and center manifolds for the modified random dynamical system $\varphi(n, \omega, x)$ under certain conditions.

Before giving the center-unstable manifold theorem, we introduce two important lemmas as follows.

Lemma 3.1. Assume that Hypotheses 2.7 and 2.8 hold. Let $\gamma(\omega)$ be a $\theta$-invariant random variable satisfying $0>\gamma(\omega)>-\beta(\omega)$. Then, $\mathbf{x}=$ $\left\{x_{n}\right\}_{n \in \mathbb{Z}^{-}} \in C_{\gamma(\omega)}^{-}$is an orbit of $\varphi(n, \omega, x)$ if and only if $\mathbf{x}=\left\{x_{n}\right\}_{n \in \mathbb{Z}^{-}}$ satisfies the following equations: 


$$
\begin{aligned}
x_{n}= & \Phi^{c u}(n, \omega) \xi-\sum_{i=n}^{-1} \Phi^{c u}\left(n-1-i, \theta^{i+1} \omega\right) F_{\rho}^{c u}\left(\theta^{i} \omega, x_{i}\right) \\
& +\sum_{i=-\infty}^{n-1} \Phi^{s}\left(n-1-i, \theta^{i+1} \omega\right) F_{\rho}^{s}\left(\theta^{i} \omega, x_{i}\right) \quad \text { for all } n \leq-1, \\
x_{0}= & \xi+\sum_{i=-\infty}^{-1} \Phi^{s}\left(-1-i, \theta^{i+1} \omega\right) F_{\rho}^{s}\left(\theta^{i} \omega, x_{i}\right),
\end{aligned}
$$

where $\xi:=x_{0}^{c u}=P^{c u} x_{0}, \Phi^{\tau}(n, \omega):=\Phi(n, \omega) P^{\tau}$ and $F_{\rho}^{\tau}(\omega, x):=$ $P^{\tau} F_{\rho}(\omega, x)$ for $\tau=c u$ and $s$.

Proof. Assume that $\mathbf{x}=\left\{x_{n}\right\}_{n \in \mathbb{Z}^{-}} \in C_{\gamma(\omega)}^{-}$is the orbit of $\varphi(n, \omega, x)$. By equation (2.3), we have, for every $k<n \leq 0$,

$$
x_{n}=\Phi\left(n-k, \theta^{k} \omega\right) x_{k}+\sum_{i=k}^{n-1} \Phi\left(n-1-i, \theta^{i+1} \omega\right) F_{\rho}\left(\theta^{i} \omega, x_{i}\right) .
$$

Let $n=0$. Then $\Phi\left(-k, \theta^{k} \omega\right)=\Phi^{-1}(k, \omega)$ yields

$$
x_{k}=\Phi(k, \omega) x_{0}-\sum_{i=k}^{-1} \Phi\left(k-1-i, \theta^{i+1} \omega\right) F_{\rho}\left(\theta^{i} \omega, x_{i}\right) .
$$

Switching index $k$ back to $n$, we obtain

$$
\begin{aligned}
& x_{n}=\Phi(n, \omega) x_{0}-\sum_{i=n}^{-1} \Phi\left(n-1-i, \theta^{i+1} \omega\right) F_{\rho}\left(\theta^{i} \omega, x_{i}\right) \\
& \text { for all } n \leq-1 .
\end{aligned}
$$

Using projection $P^{c u}$ in equation (3.2), we find that $x_{n}$ 's centerunstable component satisfies

$$
x_{n}^{c u}=\Phi^{c u}(n, \omega) x_{0}^{c u}-\sum_{i=n}^{-1} \Phi^{c u}\left(n-1-i, \theta^{i+1} \omega\right) F_{\rho}^{c u}\left(\theta^{i} \omega, x_{i}\right) .
$$

Applying the stable projection $P^{s}$ to equation (2.3), we get

$$
x_{n}^{s}=\Phi^{s}\left(n-k, \theta^{k} \omega\right) x_{k}^{s}+\sum_{i=k}^{n-1} \Phi^{s}\left(n-1-i, \theta^{i+1} \omega\right) F_{\rho}^{s}\left(\theta^{i} \omega, x_{i}\right) .
$$


Since $\left\{x_{n}\right\}_{n \in \mathbb{Z}^{-}} \in C_{\gamma(\omega)}^{-}$, it then follows from Lemma 2.12 that $\left|\Phi^{s}\left(n-k, \theta^{k} \omega\right) x_{k}^{s}\right| \leq K\left(\theta^{k} \omega\right) e^{-\beta(\omega)(n-k)} e^{\gamma(\omega) k}|x|_{\gamma(\omega)}^{-} \longrightarrow 0$ as $k \rightarrow-\infty$, where the fact that $K(\omega)$ is tempered is used. Thus, letting $k \rightarrow-\infty$ in equation (3.4), we obtain

$$
x_{n}^{s}=\sum_{i=-\infty}^{n-1} \Phi^{s}\left(n-1-i, \theta^{i+1} \omega\right) F_{\rho}^{s}\left(\theta^{i} \omega, x_{i}\right) .
$$

In particular, when $n=0$,

$$
x_{0}^{s}=\sum_{i=-\infty}^{-1} \Phi^{s}\left(-1-i, \theta^{i+1} \omega\right) F_{\rho}^{s}\left(\theta^{i} \omega, x_{i}\right) .
$$

By equations (3.3), (3.5) and (3.6), equation (3.1) is established. The converse direction follows from straightforward computation. Thus, the proof is complete.

Lemma 3.2. Assume that Hypotheses 2.7-2.14 hold. For each

$$
\gamma(\omega) \in\left[-\frac{\beta(\omega)}{2},-\frac{\beta(\omega)}{2(N+1)^{2}}\right],
$$

equation (3.1) has a unique solution $\mathbf{x}(\xi, \omega) \in C_{\gamma(\omega)}^{-}$with $x_{0}^{c u}=\xi$ for all $\xi \in E^{c u}$, and

(i) the solution $\mathbf{x}(\xi, \omega)$ is independent of

$$
\gamma(\omega) \in\left[-\frac{\beta(\omega)}{2},-\frac{\beta(\omega)}{2(N+1)^{2}}\right] ;
$$

(ii) $\mathbf{x}(\xi, \omega)$ is $\mathcal{B}\left(E^{c u}\right) \otimes \mathcal{F}$-measurable and $\mathbf{x}(\xi, \omega)$ is Lipschitz continuous in $\xi$ with

$$
\operatorname{Lip} \mathbf{x}(\cdot, \omega) \leq \frac{3 K(\omega)}{2}
$$

Furthermore, for any

$$
\gamma \in\left[-\frac{\beta(\omega)}{2(N+1)},-\frac{\beta(\omega)}{2(N+1)^{2}}\right],
$$

(iii) $\mathbf{x}(\cdot, \omega)$ is $C^{N}$ from $E^{c u}$ to $C_{N \gamma(\omega)}^{-}$with $\mathbf{x}(0, \omega)=0$ and $D_{\xi} x_{n}(0, \omega)=\Phi^{c u}(n, \omega)$ 
(iv) for each $1 \leq i \leq N$, there exists a $\theta$-invariant random variable $\zeta(\omega)=\zeta_{\gamma}(\omega)>0$ such that

$$
\left\|D_{\xi}^{i} \mathbf{x}(\xi, \omega)\right\|_{L^{i}\left(E^{c u}, C_{i \gamma(\omega)+\zeta(\omega)}^{-}\right)} \leq K_{i}(\omega),
$$

where each $K_{i}(\omega)$ is random variable tempered from above;

(v) in addition, if Hypothesis 2.15 holds, $\mathbf{x}(\cdot, \omega)$ is $C^{N, \tau(\omega)}$ from $E^{c u}$ to $C_{(N+\tau(\omega)) \gamma(\omega)}^{-}$.

Proof. First, we will prove that equation (3.1) has a unique solution $\mathbf{x}=\mathbf{x}(\xi, \omega)$ which is Lipschitz continuous with $\xi \in E^{c u}$.

Let $\mathcal{T}^{c u}(\mathbf{x}, \xi, \omega)$ denote the right hand side of equation (3.1). Set $\mathbf{y}=\left\{y_{n}\right\}_{n \in \mathbb{Z}^{-}}=: \mathcal{T}^{c u}(\mathbf{x}, \xi, \omega)$. Multiplying both sides of equation (3.1) by $e^{-\gamma(\omega) n}$, by virtue of Lemma 2.16 , we get that

$$
\begin{gathered}
e^{-\gamma(\omega) n}\left|y_{n}\right| \leq K(\omega)|\xi|+\frac{1}{3} L_{1}(\alpha, \beta)^{-1} L(\alpha, \beta, \gamma)|\mathbf{x}|_{\gamma(\omega)}^{-} \\
\text {for all } n \leq-1,
\end{gathered}
$$

where

$$
L(\alpha, \beta, \gamma):=-\frac{e^{-\gamma(\omega)}}{\alpha(\omega)+\gamma(\omega)}+\frac{e^{\beta(\omega)}}{\gamma(\omega)+\beta(\omega)}
$$

Similarly, we have

$$
\left|y_{0}\right| \leq|\xi|+\frac{1}{3} L_{1}(\alpha, \beta)^{-1} \frac{e^{\beta(\omega)}}{\gamma(\omega)+\beta(\omega)}|\mathbf{x}|_{\gamma(\omega)}^{-} .
$$

Thus, $\mathcal{T}^{c u}(\cdot, \xi, \omega)$ is a self-map on $C_{\gamma(\omega)}^{-}$.

For every $\mathbf{x}, \overline{\mathbf{x}} \in C_{\gamma(\omega)}^{-}$, we obtain

$$
\begin{aligned}
\left|y_{n}-\bar{y}_{n}\right| \leq & \sum_{i=n}^{-1}\left\|\Phi^{c u}\left(n-1-i, \theta^{i+1} \omega\right)\right\| \\
& \times\left|F_{\rho}^{c u}\left(\theta^{i} \omega, x_{i}\right)-F_{\rho}^{c u}\left(\theta^{i} \omega, \bar{x}_{i}\right)\right|+\sum_{i=-\infty}^{n-1}\left\|\Phi^{s}\left(n-1-i, \theta^{i+1} \omega\right)\right\| \\
& \times\left|F_{\rho}^{s}\left(\theta^{i} \omega, x_{i}\right)-F_{\rho}^{s}\left(\theta^{i} \omega, \bar{x}_{i}\right)\right| \quad \text { for all } n \leq-1,
\end{aligned}
$$


where $\overline{\mathbf{y}}=\left\{\bar{y}_{n}\right\}_{n \in \mathbb{Z}^{-}}:=\mathcal{T}^{c u}(\overline{\mathbf{x}}, \xi, \omega)$, which implies

$$
\left|y_{n}-\bar{y}_{n}\right| e^{-\gamma(\omega) n} \leq \frac{1}{3} L_{1}(\alpha, \beta)^{-1} L(\alpha, \beta, \gamma)|\mathbf{x}-\overline{\mathbf{x}}|_{\gamma(\omega)}^{-} .
$$

Similarly,

$$
\left|y_{0}-\bar{y}_{0}\right| \leq \frac{1}{3} L_{1}(\alpha, \beta)^{-1} \frac{e^{\beta(\omega)}}{\gamma(\omega)+\beta(\omega)}|\mathbf{x}-\overline{\mathbf{x}}|_{\gamma(\omega)}^{-} .
$$

Then, we obtain that

$$
\left|\mathcal{T}^{c u}(\mathbf{x}, \xi, \omega)-\mathcal{T}^{c u}(\overline{\mathbf{x}}, \xi, \omega)\right|_{\gamma(\omega)}^{-} \leq \frac{1}{3} L_{1}(\alpha, \beta)^{-1} L(\alpha, \beta, \gamma)|\mathbf{x}-\overline{\mathbf{x}}|_{\gamma(\omega)}^{-} .
$$

Since

$$
L(\alpha, \beta, \gamma) \leq L_{1}(\alpha, \beta) \quad \text { for all } \gamma \in\left[-\frac{\beta(\omega)}{2},-\frac{\beta(\omega)}{2(N+1)^{2}}\right],
$$

it then follows that $\mathcal{T}^{c u}(\cdot, \xi, \omega)$ is a uniform contraction with respect to the parameter $\xi$. By the contraction mapping principle, we have that, for each $\xi \in E^{c u}, \mathcal{T}^{c u}(\cdot, \xi, \omega)$ has a unique fixed point $\mathbf{x}(\xi, \omega)$. Obviously, $\mathbf{x}(0, \omega)=0$. Moreover, for every $\xi, \xi_{0} \in E^{c u}$ and $n \leq-1$, we have

$$
\begin{aligned}
\left|x_{n}(\xi, \omega)-x_{n}\left(\xi_{0}, \omega\right)\right| e^{-\gamma(\omega) n} \leq K(\omega)\left|\xi-\xi_{0}\right| \\
+\frac{1}{3} L_{1}(\alpha, \beta)^{-1} L(\alpha, \beta, \gamma)\left|\mathbf{x}(\xi, \omega)-\mathbf{x}\left(\xi_{0}, \omega\right)\right|_{\gamma(\omega)}^{-} .
\end{aligned}
$$

Hence,

$$
\left|\mathbf{x}(\xi, \omega)-\mathbf{x}\left(\xi_{0}, \omega\right)\right|_{\gamma(\omega)}^{-} \leq \frac{3 K(\omega)}{2}\left|\xi-\xi_{0}\right| .
$$

Since $\mathbf{x}(\xi, \omega)$ is the $\omega$-wise limit of iteration of contraction mapping $\mathcal{T}^{c u}$ starting at 0 and $\mathcal{T}^{c u}$ maps a $\mathcal{F}$-measurable function to a measurable function, $\mathbf{x}(\xi, \omega)$ is $\mathcal{F}$-measurable. Besides, equation (3.9) shows that, for any $\omega \in \Omega, \mathbf{x}(\cdot, \omega)$ is Lipschitz continuous. By [7, Lemma III.14], $\mathbf{x}(\xi, \omega)$ is $\mathcal{B}\left(E^{c u}\right) \otimes \mathcal{F}$-measurable.

Noting that

$$
-\frac{\beta(\omega)}{2} \leq \gamma^{\prime}(\omega) \leq \gamma^{\prime \prime}(\omega) \leq-\frac{\beta(\omega)}{2(N+1)^{2}},
$$


we have that $C_{\gamma^{\prime \prime}(\omega)}^{-} \subset C_{\gamma^{\prime}(\omega)}^{-}$. Then, a fixed point in $C_{\gamma^{\prime \prime}(\omega)}^{-}$must be in $C_{\gamma^{\prime}(\omega)}^{-}$. This implies that $\mathbf{x}(\xi, \omega)$ is independent of

$$
\gamma(\omega) \in\left[-\frac{\beta(\omega)}{2},-\frac{\beta(\omega)}{2(N+1)^{2}}\right]
$$

For each

$$
\gamma(\omega) \in\left[-\frac{\beta(\omega)}{2(N+1)},-\frac{\beta(\omega)}{2(N+1)^{2}}\right],
$$

we choose a $\theta$-invariant random variable $\zeta(\omega)>0$ such that

$$
\begin{gathered}
-\frac{\beta(\omega)}{2}<\ell \gamma(\omega)+\zeta(\omega)<\ell \gamma(\omega)+2 \zeta(\omega)<-\frac{\beta(\omega)}{2(N+1)^{2}} \\
\text { for all } \ell=1, \ldots, N
\end{gathered}
$$

Next we prove that $\mathbf{x}(\cdot, \omega) \in C^{N}$ by induction. In order to fulfill this goal, we first prove that $\mathbf{x}(\cdot, \omega)$ is differentiable from $E^{c u}$ to $C_{\gamma(\omega)+\zeta(\omega)}^{-}$ and then show that $D_{\xi} \mathbf{x}(\cdot, \omega): E^{c u} \rightarrow L\left(E^{c u}, C_{\gamma(\omega)}^{-}\right)$is continuous.

Define the linear operator $\mathcal{G}: C_{\gamma(\omega)+\zeta(\omega)}^{-} \rightarrow C_{\gamma(\omega)+\zeta(\omega)}^{-}$by

$$
\begin{aligned}
&(\mathcal{G} v)_{n}=-\sum_{i=n}^{-1} \Phi^{c u}\left(n-1-i, \theta^{i+1} \omega\right) D_{x} F_{\rho}^{c u}\left(\theta^{i} \omega, x_{i}\left(\xi_{0}, \omega\right)\right) v_{i} \\
&+\sum_{i=-\infty}^{n-1} \Phi^{s}\left(n-1-i, \theta^{i+1} \omega\right) D_{x} F_{\rho}^{s}\left(\theta^{i} \omega, x_{i}\left(\xi_{0}, \omega\right)\right) v_{i} \\
& \quad \text { for all } n \leq-1, \\
&(\mathcal{G} v)_{0}=\sum_{i=-\infty}^{-1} \Phi^{s}\left(-1-i, \theta^{i+1} \omega\right) D_{x} F_{\rho}^{s}\left(\theta^{i} \omega, x_{i}\left(\xi_{0}, \omega\right)\right) v_{i} .
\end{aligned}
$$

It is clear that $\mathcal{G}$ is a bounded linear operator on $C_{\gamma(\omega)+\zeta(\omega)}^{-}$with the norm

$$
\|\mathcal{G}\| \leq \frac{1}{3} L_{1}(\alpha, \beta)^{-1} L(\alpha, \beta, \gamma+\zeta) .
$$

By equation (3.8), we see that Id $-\mathcal{G}$ has a bounded inverse in 


$$
\begin{aligned}
C_{\gamma(\omega)+}^{-} & \zeta(\omega) \text {. Let } \\
I_{n}= & -\sum_{i=n}^{-1} \Phi^{c u}\left(n-1-i, \theta^{i+1} \omega\right)\left(F_{\rho}^{c u}\left(\theta^{i} \omega, x_{i}(\xi, \omega)\right)-F_{\rho}^{c u}\left(\theta^{i} \omega, x_{i}\left(\xi_{0}, \omega\right)\right)\right. \\
& \left.-D_{x} F_{\rho}^{c u}\left(\theta^{i} \omega, x_{i}\left(\xi_{0}, \omega\right)\right)\left(x_{i}(\xi, \omega)-x_{i}\left(\xi_{0}, \omega\right)\right)\right) \\
& +\sum_{i=-\infty}^{n-1} \Phi^{s}\left(n-1-i, \theta^{i+1} \omega\right)\left(F_{\rho}^{s}\left(\theta^{i} \omega, x_{i}(\xi, \omega)\right)-F_{\rho}^{s}\left(\theta^{i} \omega, x_{i}\left(\xi_{0}, \omega\right)\right)\right. \\
& \left.-D_{x} F_{\rho}^{s}\left(\theta^{i} \omega, x_{i}\left(\xi_{0}, \omega\right)\right)\left(x_{i}(\xi, \omega)-x_{i}\left(\xi_{0}, \omega\right)\right)\right) \quad \text { for all } n \leq-1, \\
I_{0}= & \sum_{i=-\infty}^{-1} \Phi^{s}\left(-1-i, \theta^{i+1} \omega\right)\left(F_{\rho}^{s}\left(\theta^{i} \omega, x_{i}(\xi, \omega)\right)-F_{\rho}^{s}\left(\theta^{i} \omega, x_{i}\left(\xi_{0}, \omega\right)\right)\right. \\
& \left.-D_{x} F_{\rho}^{s}\left(\theta^{i} \omega, x_{i}\left(\xi_{0}, \omega\right)\right)\left(x_{i}(\xi, \omega)-x_{i}\left(\xi_{0}, \omega\right)\right)\right) .
\end{aligned}
$$

We denote the bounded operator $\mathcal{L}^{c u}: E^{c u} \rightarrow C_{\gamma(\omega)+\zeta(\omega)}^{-}$by $\left(\mathcal{L}^{c u} \xi\right)_{n}:=$ $\Phi^{c u}(n, \omega) \xi$. Set $I:=\left\{I_{n}\right\}_{n \in \mathbb{Z}^{-}}$. Next, we prove the claim $|I|_{\gamma(\omega)+\zeta(\omega)}^{-}=$ $o\left(\left|\xi-\xi_{0}\right|\right)$. Divide $e^{-(\gamma(\omega)+\zeta(\omega)) n} I_{n}$ by a sum of four terms, i.e., $e^{-(\gamma(\omega)+\zeta(\omega)) n} I_{n}=I_{n}^{\prime}+I_{n}^{\prime \prime}+I_{n}^{\prime \prime \prime}+I_{n}^{\prime \prime \prime \prime}$, where

$$
\begin{aligned}
I_{n}^{\prime}= & -e^{-(\gamma(\omega)+\zeta(\omega)) n} \sum_{i=n}^{\bar{N}-1} \Phi^{c u}\left(n-1-i, \theta^{i+1} \omega\right) \\
& \times\left(F_{\rho}^{c u}\left(\theta^{i} \omega, x_{i}(\xi, \omega)\right)-F_{\rho}^{c u}\left(\theta^{i} \omega, x_{i}\left(\xi_{0}, \omega\right)\right)\right. \\
& \left.-D_{x} F_{\rho}^{c u}\left(\theta^{i} \omega, x_{i}\left(\xi_{0}, \omega\right)\right)\left(x_{i}(\xi, \omega)-x_{i}\left(\xi_{0}, \omega\right)\right)\right)
\end{aligned}
$$

for $n<\bar{N} \leq-1$ and $I_{n}^{\prime}=0$ for $0 \geq n \geq \bar{N}$,

$$
\begin{aligned}
I_{n}^{\prime \prime}= & -e^{-(\gamma(\omega)+\zeta(\omega)) n} \sum_{i=\bar{N}}^{-1} \Phi^{c u}\left(n-1-i, \theta^{i+1} \omega\right) \\
& \times\left(F_{\rho}^{c u}\left(\theta^{i} \omega, x_{i}(\xi, \omega)\right)-F_{\rho}^{c u}\left(\theta^{i} \omega, x_{i}\left(\xi_{0}, \omega\right)\right)\right.
\end{aligned}
$$




$$
\left.-D_{x} F_{\rho}^{c u}\left(\theta^{i} \omega, x_{i}\left(\xi_{0}, \omega\right)\right)\left(x_{i}(\xi, \omega)-x_{i}\left(\xi_{0}, \omega\right)\right)\right)
$$

for $n<\bar{N} \leq-1$ and change $\bar{N}$ to $n$ if $-1 \geq n \geq \bar{N}$ and $I_{n}^{\prime \prime}=0$ if $n=0$,

$$
\begin{aligned}
I_{n}^{\prime \prime \prime}= & e^{-(\gamma(\omega)+\zeta(\omega)) n} \sum_{i=-\infty}^{\overline{\bar{N}}-1} \Phi^{s}\left(n-1-i, \theta^{i+1} \omega\right) \\
& \times\left(F_{\rho}^{s}\left(\theta^{i} \omega, x_{i}(\xi, \omega)\right)-F_{\rho}^{s}\left(\theta^{i} \omega, x_{i}\left(\xi_{0}, \omega\right)\right)\right. \\
& \left.-D_{x} F_{\rho}^{s}\left(\theta^{i} \omega, x_{i}\left(\xi_{0}, \omega\right)\right)\left(x_{i}(\xi, \omega)-x_{i}\left(\xi_{0}, \omega\right)\right)\right)
\end{aligned}
$$

for $\overline{\bar{N}}<n \leq 0$ and change $\overline{\bar{N}}-1$ to $n-1$ for $\overline{\bar{N}} \geq n$, and

$$
\begin{aligned}
I_{n}^{\prime \prime \prime \prime}= & e^{-(\gamma(\omega)+\zeta(\omega)) n} \sum_{i=\overline{\bar{N}}}^{n-1} \Phi^{s}\left(n-1-i, \theta^{i+1} \omega\right) \\
& \times\left(F_{\rho}^{s}\left(\theta^{i} \omega, x_{i}(\xi, \omega)\right)-F_{\rho}^{s}\left(\theta^{i} \omega, x_{i}\left(\xi_{0}, \omega\right)\right)\right. \\
& \left.-D_{x} F_{\rho}^{s}\left(\theta^{i} \omega, x_{i}\left(\xi_{0}, \omega\right)\right)\left(x_{i}(\xi, \omega)-x_{i}\left(\xi_{0}, \omega\right)\right)\right)
\end{aligned}
$$

for $\overline{\bar{N}}<n \leq 0$ and $I_{n}^{\prime \prime \prime \prime}=0$ for $\overline{\bar{N}} \geq n$. Here, $-\bar{N}$ and $-\overline{\bar{N}}$ are large positive numbers to be chosen later. We first observe that, for $n<\bar{N} \leq-1$,

$$
\begin{aligned}
\left|I_{n}^{\prime}\right| \leq & e^{\alpha(\omega)+(-\alpha(\omega)-\gamma(\omega)-\zeta(\omega)) n} \\
& +\sum_{i=n}^{\bar{N}-1} 20 K\left(\theta^{i+1} \omega\right) \widetilde{B}_{2}\left(\theta^{i} \omega\right) \rho\left(\theta^{i} \omega\right) e^{(\gamma(\omega)+2 \zeta(\omega)+\alpha(\omega)) i} \\
& \cdot\left|\mathbf{x}(\xi, \omega)-\mathbf{x}\left(\xi_{0}, \omega\right)\right|_{\gamma(\omega)+2 \zeta(\omega)}^{-} \\
\leq & \frac{2 L_{1}(\alpha, \beta)^{-1}}{-3(\alpha(\omega)+\gamma(\omega)+2 \zeta(\omega))} \\
& \times e^{-\gamma(\omega)-2 \zeta(\omega)+\zeta(\omega) \bar{N}}\left|\mathbf{x}(\xi, \omega)-\mathbf{x}\left(\xi_{0}, \omega\right)\right|_{\gamma(\omega)+2 \zeta(\omega)}^{-} \\
\leq & \frac{2 L_{1}(\alpha, \beta)^{-1}}{-3(\alpha(\omega)+\gamma(\omega)+2 \zeta(\omega))}
\end{aligned}
$$




$$
\times e^{-\gamma(\omega)-2 \zeta(\omega)+\zeta(\omega) \bar{N}} \cdot \frac{3 K(\omega)}{2}\left|\xi-\xi_{0}\right|
$$

where the last inequality follows from equation (3.9). Choose $-\bar{N}$ large enough so that

$$
\sup _{n \in \mathbb{Z}^{-}}\left|I_{n}^{\prime}\right| \leq \frac{\varepsilon}{4}\left|\xi-\xi_{0}\right|
$$

Fix $\bar{N}$. We have that, for every $n \in \mathbb{Z}^{-}$,

$$
\begin{aligned}
\left|I_{n}^{\prime \prime}\right| \leq & \left|\mathbf{x}(\xi, \omega)-\mathbf{x}\left(\xi_{0}, \omega\right)\right|_{\gamma(\omega)}^{-} \\
& \times\left(e^{\alpha(\omega)+(-\alpha(\omega)-\gamma(\omega)-\zeta(\omega)) n} \sum_{i=\bar{N}}^{-1} K\left(\theta^{i+1} \omega\right) e^{(\gamma(\omega)+\alpha(\omega)) i}\right. \\
& \quad \times \int_{0}^{1} \| D_{x} F_{\rho}^{c u}\left(\theta^{i} \omega, s x_{i}(\xi, \omega)+(1-s) x_{i}\left(\xi_{0}, \omega\right)\right) \\
& \left.-D_{x} F_{\rho}^{c u}\left(\theta^{i} \omega, x_{i}\left(\xi_{0}, \omega\right)\right) \| d s\right) .
\end{aligned}
$$

Since $F_{\rho}(\omega, x) \in C^{N} N \geq 2$, for each $\varepsilon>0$, there exists $\delta_{i}=\delta_{i}(\omega)$ such that, if $\left|\xi-\xi_{0}\right|<\delta_{i}$,

$$
\begin{gathered}
\| D_{x} F_{\rho}^{c u}\left(\theta^{i} \omega, s x_{i}(\xi, \omega)+(1-s) x_{i}\left(\xi_{0}, \omega\right)\right) \\
-D_{x} F_{\rho}^{c u}\left(\theta^{i} \omega, x_{i}\left(\xi_{0}, \omega\right)\right) \| \\
\leq \frac{\varepsilon}{-6 \bar{N} K(\omega) K\left(\theta^{i+1} \omega\right) e^{(\gamma(\omega)+\alpha(\omega)) i} e^{\alpha(\omega)}} \\
\text { for } i=\bar{N}, \ldots,-1 .
\end{gathered}
$$

Letting $\bar{\delta}=\min _{\bar{N}<i<-1}\left\{\delta_{i}\right\}$ and recalling equation (3.9), we obtain as $\left|\xi-\xi_{0}\right|<\bar{\delta}$

$$
\sup _{n \in \mathbb{Z}^{-}}\left|I_{n}^{\prime \prime}\right| \leq \frac{\varepsilon}{4}\left|\xi-\xi_{0}\right|
$$

Similarly, we choose $-\overline{\bar{N}}$ large enough so that

$$
\sup _{n \in \mathbb{Z}^{-}}\left|I_{n}^{\prime \prime \prime}\right| \leq \frac{\varepsilon}{4}\left|\xi-\xi_{0}\right|
$$


and there exists $\overline{\bar{\delta}}=\overline{\bar{\delta}}(\omega)$ such that, as $\left|\xi-\xi_{0}\right|<\overline{\bar{\delta}}$,

$$
\sup _{n \in \mathbb{Z}^{-}}\left|I_{n}^{\prime \prime \prime \prime}\right| \leq \frac{\varepsilon}{4}\left|\xi-\xi_{0}\right| .
$$

Taking $\widetilde{\delta}:=\min \{\bar{\delta}, \overline{\bar{\delta}}\}$ and combining (3.11)-(3.14), we obtain that

$$
|I|_{\gamma(\omega)+\zeta(\omega)}^{-} \leq \varepsilon\left|\xi-\xi_{0}\right| \quad \text { for }\left|\xi-\xi_{0}\right|<\widetilde{\delta}
$$

Then the claim is true. Thus,

$$
\mathbf{x}(\xi, \omega)-\mathbf{x}\left(\xi_{0}, \omega\right)=(\operatorname{Id}-\mathcal{G})^{-1} \mathcal{L}^{c u}\left(\xi-\xi_{0}\right)+o\left(\left|\xi-\xi_{0}\right|\right) .
$$

This yields $\mathbf{x}(\xi, \omega)$ is differentiable in $\xi$ and

$$
D_{\xi} \mathbf{x}(\xi, \omega) \in L\left(E^{c u}, C_{\gamma(\omega)+\zeta(\omega)}^{-}\right)
$$

By equation (3.1), we find $D_{\xi} \mathbf{x}(\cdot, \omega): E^{c u} \rightarrow L\left(E^{c u}, C_{\gamma(\omega)+\zeta(\omega)}^{-}\right)$ satisfies

$$
\begin{aligned}
& D_{\xi} x_{n}(\xi, \omega)= \Phi^{c u}(n, \omega) \\
&-\sum_{i=n}^{-1} \Phi^{c u}\left(n-1-i, \theta^{i+1} \omega\right) D_{x} F_{\rho}^{c u}\left(\theta^{i} \omega, x_{i}(\xi, \omega)\right) D_{\xi} x_{i}(\xi, \omega) \\
&+\sum_{i=-\infty}^{n-1} \Phi^{s}\left(n-1-i, \theta^{i+1} \omega\right) D_{x} F_{\rho}^{s}\left(\theta^{i} \omega, x_{i}(\xi, \omega)\right) D_{\xi} x_{i}(\xi, \omega) \\
&(3.15) \quad \text { for all } n \leq-1, \\
& D_{\xi} x_{0}(\xi, \omega)=\operatorname{Id}+\sum_{i=-\infty}^{-1} \Phi^{s}\left(-1-i, \theta^{i+1} \omega\right) D_{x} F_{\rho}^{s}\left(\theta^{i} \omega, x_{i}(\xi, \omega)\right) D_{\xi} x_{i}(\xi, \omega) .
\end{aligned}
$$

Furthermore,

$$
\left\|D_{\xi} \mathbf{x}(\xi, \omega)\right\|_{L\left(E^{c u}, C_{\gamma(\omega)+\zeta(\omega)}^{-}\right.} \leq \frac{3 K(\omega)}{2} .
$$

By the contraction mapping principle, $D_{\xi} \mathbf{x}(\xi, \omega)$ is a unique solution of equation (3.15). Recalling that $D_{x} F(\omega, 0)=0$ and $\mathbf{x}(0, \omega)=0$, we have $D_{\xi} x_{n}(0, \omega)=\Phi^{c u}(n, \omega)$.

To prove that $D_{\xi} \mathbf{x}(\xi, \omega)$ is continuous with respect to $\xi$, we define the operator $\mathcal{G}_{1}: L\left(E^{c u}, C_{\gamma(\omega)}^{-}\right) \rightarrow L\left(E^{c u}, C_{\gamma(\omega)}^{-}\right)$by the right hand side 
of equation (3.10) with $\xi$ in place of $\xi_{0}$. Let $H:=\left\{H_{n}\right\}_{n \in \mathbb{Z}^{-}}$, where

$$
\begin{aligned}
H_{n}= & -\sum_{i=n}^{-1} \Phi^{c u}\left(n-1-i, \theta^{i+1} \omega\right)\left(D_{x} F_{\rho}^{c u}\left(\theta^{i} \omega, x_{i}(\xi, \omega)\right)\right. \\
& \left.-D_{x} F_{\rho}^{c u}\left(\theta^{i} \omega, x_{i}\left(\xi_{0}, \omega\right)\right)\right) D_{\xi} x_{i}\left(\xi_{0}, \omega\right) \\
& +\sum_{i=-\infty}^{n-1} \Phi^{s}\left(n-1-i, \theta^{i+1} \omega\right)\left(D_{x} F_{\rho}^{s}\left(\theta^{i} \omega, x_{i}(\xi, \omega)\right)\right. \\
& \left.-D_{x} F_{\rho}^{s}\left(\theta^{i} \omega, x_{i}\left(\xi_{0}, \omega\right)\right)\right) D_{\xi} x_{i}\left(\xi_{0}, \omega\right) \quad \text { for all } n \leq-1, \\
H_{0}= & \sum_{i=-\infty}^{-1} \Phi^{s}\left(-1-i, \theta^{i+1} \omega\right)\left(D_{x} F_{\rho}^{s}\left(\theta^{i} \omega, x_{i}(\xi, \omega)\right)\right. \\
& \left.-D_{x} F_{\rho}^{s}\left(\theta^{i} \omega, x_{i}\left(\xi_{0}, \omega\right)\right)\right) D_{\xi} x_{i}\left(\xi_{0}, \omega\right) .
\end{aligned}
$$

Then, we have

$$
D_{\xi} \mathbf{x}(\xi, \omega)-D_{\xi} \mathbf{x}\left(\xi_{0}, \omega\right)=\mathcal{G}_{1}\left(D_{\xi} \mathbf{x}(\xi, \omega)-D_{\xi} \mathbf{x}\left(\xi_{0}, \omega\right)\right)+H .
$$

Using the same procedure as for $I$, we get $\|H\|_{L\left(E^{c u}, C_{\gamma(\omega)}^{-}\right)}=o(1)$ as $\xi \rightarrow \xi_{0}$. On the other hand, it follows from equation (3.10) that

$$
\left\|\mathcal{G}_{1}\right\| \leq \frac{1}{3} L_{1}(\alpha, \beta)^{-1} L(\alpha, \beta, \gamma)
$$

Equation (3.8) implies Id $-\mathcal{G}_{1}$ has a bounded inverse in $L\left(E^{c u}, C_{\gamma(\omega)}^{-}\right)$. In view of equation (3.16) we see that $D_{\xi} \mathbf{x}(\xi, \omega)$ is continuous with respect to $\xi$.

Now, we use inductive assumption to prove that $\mathbf{x}(\cdot, \omega)$ is $C^{j}$ from $E^{c u}$ to $C_{j \gamma(\omega)}^{-}$for all $1 \leq j \leq m-1,2 \leq m \leq N$. Assume that there exist random variables tempered from above $K_{j}(\omega)$ such that

$$
\left\|D_{\xi}^{j} \mathbf{x}(\xi, \omega)\right\|_{L^{j}\left(E^{c u}, C_{j \gamma(\omega)+\zeta(\omega)}^{-}\right)} \leq K_{j}(\omega)
$$

and prove it for $j=m$. By simple computation, when $m \geq 3$, $D_{\xi}^{m-1} \mathbf{x}(\cdot, \omega)$ satisfies the following:

$$
D_{\xi}^{m-1} x_{n}(\xi, \omega)=-\sum_{i=n}^{-1} \Phi^{c u}\left(n-1-i, \theta^{i+1} \omega\right) D_{x} F_{\rho}^{c u}\left(\theta^{i} \omega, x_{i}(\xi, \omega)\right)
$$




$$
\times D_{\xi}^{m-1} x_{i}(\xi, \omega)+\sum_{i=-\infty}^{n-1} \Phi^{s}\left(n-1-i, \theta^{i+1} \omega\right) D_{x} F_{\rho}^{s}\left(\theta^{i} \omega, x_{i}(\xi, \omega)\right)
$$

$$
\begin{gathered}
\times D_{\xi}^{m-1} x_{i}(\xi, \omega)-\sum_{i=n}^{-1} \Phi^{c u}\left(n-1-i, \theta^{i+1} \omega\right) R_{m-1, i}^{c u}(\xi, \omega) \\
+\sum_{i=-\infty}^{n-1} \Phi^{s}\left(n-1-i, \theta^{i+1} \omega\right) R_{m-1, i}^{s}(\xi, \omega) \\
\quad \text { for all } n \leq-1, \\
D_{\xi}^{m-1} x_{0}(\xi, \omega)=\sum_{i=-\infty}^{-1} \Phi^{s}\left(-1-i, \theta^{i+1} \omega\right) D_{x} F_{\rho}^{s}\left(\theta^{i} \omega, x_{i}(\xi, \omega)\right) \\
\times D_{\xi}^{m-1} x_{i}(\xi, \omega)+\sum_{i=-\infty}^{-1} \Phi^{s}\left(-1-i, \theta^{i+1} \omega\right) R_{m-1, i}^{s}(\xi, \omega),
\end{gathered}
$$

where

$R_{m-1, i}^{\tau}(\xi, \omega)=\sum_{l=0}^{m-3}\left(\begin{array}{c}m-2 \\ l\end{array}\right) D_{\xi}^{m-2-l}\left(D_{x} F_{\rho}^{\tau}\left(\theta^{i} \omega, x_{i}(\xi, \omega)\right)\right) D_{\xi}^{l+1} x_{i}(\xi, \omega)$

for $\tau=c u, s$. In the case of $m=2$, see equation (3.15). We only prove $m \geq 3$ for brevity. The proof for $m=2$ is similar. By the chain rule, we obtain that each term in $R_{m-1, i}^{\tau}(\xi, \omega)$ contains factors $D_{x}^{l_{1}} F_{\rho}^{\tau}\left(\theta^{i} \omega, x_{i}(\xi, \omega)\right)$ for some $2 \leq l_{1} \leq m-1$, and at least two derivatives $D_{\xi}^{l_{2}} x_{i}(\xi, \omega)$ and $D_{\xi}^{l_{3}} x_{i}(\xi, \omega)$ for some $l_{2}, l_{3} \in\{1, \ldots, m-2\}$. Since $D_{\xi}^{l} \mathbf{x}(\xi, \omega) \in C_{l \gamma(\omega)}^{-}$for $l=1, \ldots, m-1$ and $F$ is $C^{N}, R_{m-1, i}^{c u}(\cdot, \omega)$ : $E^{c u} \rightarrow L^{m-1}\left(E^{c u}, E^{c u}\right)$ and $R_{m-1, i}^{s}(\cdot, \omega): E^{c u} \rightarrow L^{m-1}\left(E^{c u}, E^{s}\right)$ are $C^{1}$. Furthermore, we have

$$
\left\|D_{\xi} R_{m-1, i}^{\tau}(\xi, \omega)\right\| \leq \bar{B}_{m}\left(\theta^{i} \omega\right) \widetilde{K}_{m}(\omega) e^{(m \gamma(\omega)+2 \zeta(\omega)) i} \quad \text { for } \tau=c u, s,
$$

where $\bar{B}_{m}(\omega)=\max _{1 \leq i \leq m} B_{i}(\omega)$ and $\widetilde{K}_{m}(\omega)$ is the $m$ th-order polynomial of $K_{1}(\omega), \ldots, K_{m-1}(\omega)$ with positive integer coefficients that are tempered from above. Let $J(\xi, \omega):=\left\{J_{n}(\xi, \omega)\right\}_{n \in \mathbb{Z}^{-}}$, where

$$
J_{n}(\xi, \omega)=-\sum_{i=n}^{-1} \Phi^{c u}\left(n-1-i, \theta^{i+1} \omega\right) R_{m-1, i}^{c u}(\xi, \omega)
$$




$$
+\sum_{i=-\infty}^{n-1} \Phi^{s}\left(n-1-i, \theta^{i+1} \omega\right) R_{m-1, i}^{s}(\xi, \omega) \quad \text { for all } n \leq-1,
$$

$$
J_{0}(\xi, \omega)=\sum_{i=-\infty}^{-1} \Phi^{s}\left(-1-i, \theta^{i+1} \omega\right) R_{m-1, i}^{s}(\xi, \omega) .
$$

Note that, for $n \leq i \leq-1$,

$$
\begin{aligned}
& \| \Phi^{c u}\left(n-1-i, \theta^{i+1} \omega\right) D_{\xi} R_{m-1, i}^{c u}(\xi, \omega) \| \\
& \leq\left(K\left(\theta^{i+1} \omega\right) \bar{B}_{m}\left(\theta^{i} \omega\right) e^{\zeta(\omega) i}\right) \\
& \quad \times \widetilde{K}_{m}(\omega) e^{-\alpha(\omega)(n-1-i)} e^{(m \gamma(\omega)+\zeta(\omega)) i}
\end{aligned}
$$

and, for $i \leq n-1 \leq-1$,

$$
\begin{aligned}
\| \Phi^{s}(n-1- & \left.i, \theta^{i+1} \omega\right) D_{\xi} R_{m-1, i}^{s}(\xi, \omega) \| \\
\leq & \left(K\left(\theta^{i+1} \omega\right) \bar{B}_{m}\left(\theta^{i} \omega\right) e^{\zeta(\omega) i}\right) \\
& \times \widetilde{K}_{m}(\omega) e^{-\beta(\omega)(n-1-i)} e^{(m \gamma(\omega)+\zeta(\omega)) i}
\end{aligned}
$$

Since $K\left(\theta^{i+1} \omega\right) \bar{B}_{m}\left(\theta^{i} \omega\right) e^{\zeta(\omega) i}$ is bounded by a tempered-from-above random variable $K_{*}(\omega)$ independent of $i$ (see Lemma 2.13), by equations (3.19)-(3.21), we get that $J_{n}(\xi, \omega)$ in $\xi$ is $C^{1}$ for all $n \leq 0$ and

$$
\left\|D_{\xi} J_{n}(\xi, \omega)\right\| e^{-(m \gamma(\omega)+\zeta(\omega)) n} \leq K_{*}(\omega) \widetilde{K}_{m}(\omega) L(\alpha, \beta, m \gamma+\zeta) .
$$

We consider a linear operator $\mathcal{G}$ on $L^{m-1}\left(E^{c u}, C_{m \gamma(\omega)+\zeta(\omega)}^{-}\right)$that is defined by equation (3.10). Let $I^{m}:=\left\{I_{n}^{m}\right\}_{n \in \mathbb{Z}^{-}}$, where

$$
\begin{aligned}
I_{n}^{m}= & -\sum_{i=n}^{-1} \Phi^{c u}\left(n-1-i, \theta^{i+1} \omega\right)\left(D_{x} F_{\rho}^{c u}\left(\theta^{i} \omega, x_{i}(\xi, \omega)\right)\right. \\
& \left.-D_{x} F_{\rho}^{c u}\left(\theta^{i} \omega, x_{i}\left(\xi_{0}, \omega\right)\right)\right) D_{\xi}^{m-1} x_{i}(\xi, \omega) \\
& +\sum_{i=-\infty}^{n-1} \Phi^{s}\left(n-1-i, \theta^{i+1} \omega\right)\left(D_{x} F_{\rho}^{s}\left(\theta^{i} \omega, x_{i}(\xi, \omega)\right)\right. \\
& \left.-D_{x} F_{\rho}^{s}\left(\theta^{i} \omega, x_{i}\left(\xi_{0}, \omega\right)\right)\right) D_{\xi}^{m-1} x_{i}(\xi, \omega)+R_{n}^{m}
\end{aligned}
$$


for all $n \leq-1$,

$$
\begin{aligned}
I_{0}^{m}= & \sum_{i=-\infty}^{-1} \Phi^{s}\left(-1-i, \theta^{i+1} \omega\right)\left(D_{x} F_{\rho}^{s}\left(\theta^{i} \omega, x_{i}(\xi, \omega)\right)\right. \\
& \left.-D_{x} F_{\rho}^{s}\left(\theta^{i} \omega, x_{i}\left(\xi_{0}, \omega\right)\right)\right) D_{\xi}^{m-1} x_{i}(\xi, \omega)+R_{0}^{m},
\end{aligned}
$$

and

$$
\begin{aligned}
R_{n}^{m}= & \sum_{i=n}^{-1} \Phi^{u}\left(n-1-i, \theta^{i+1} \omega\right) D_{x x} F_{\rho}^{c u}\left(\theta^{i} \omega, x_{i}\left(\xi_{0}, \omega\right)\right) \\
& \times\left(x_{i}(\xi, \omega)-x_{i}\left(\xi_{0}, \omega\right)\right) D_{\xi}^{m-1} x_{i}(\xi, \omega) \\
- & \sum_{i=-\infty}^{n-1} \Phi^{s}\left(n-1-i, \theta^{i+1} \omega\right) D_{x x} F_{\rho}^{s}\left(\theta^{i} \omega, x_{i}\left(\xi_{0}, \omega\right)\right) \\
& \times\left(x_{i}(\xi, \omega)-x_{i}\left(\xi_{0}, \omega\right)\right) D_{\xi}^{m-1} x_{i}(\xi, \omega) \quad \text { for all } n \leq-1, \\
R_{0}^{m}=- & \sum_{i=-\infty}^{-1} \Phi^{s}\left(n-1-i, \theta^{i+1} \omega\right) D_{x x} F_{\rho}^{s}\left(\theta^{i} \omega, x_{i}\left(\xi_{0}, \omega\right)\right) \\
& \times\left(x_{i}(\xi, \omega)-x_{i}\left(\xi_{0}, \omega\right)\right) D_{\xi}^{m-1} x_{i}(\xi, \omega) .
\end{aligned}
$$

Let $R^{m}:=\left\{R_{n}^{m}\right\}_{n \in \mathbb{Z}^{-}}$. By equation (3.18), we have

$$
\begin{aligned}
D_{\xi}^{m-1} \mathbf{x}(\xi, \omega)-D_{\xi}^{m-1} \mathbf{x}\left(\xi_{0}, \omega\right) & \\
-\mathcal{G}\left(D_{\xi}^{m-1} \mathbf{x}(\xi, \omega)\right. & \left.-D_{\xi}^{m-1} \mathbf{x}\left(\xi_{0}, \omega\right)\right) \\
& =J(\xi, \omega)-J\left(\xi_{0}, \omega\right)+I^{m}-R^{m} .
\end{aligned}
$$

Since $\|\mathcal{G}\| \leq 1 / 3$, the inverse operator $(\operatorname{Id}-\mathcal{G})^{-1}$ exists in $L^{m-1}\left(E^{c u}\right.$, $C_{m \gamma(\omega)+\zeta(\omega)}^{-}$). Then equation (3.22) is equivalent to

$$
\begin{aligned}
D_{\xi}^{m-1} \mathbf{x}(\xi, \omega)-D_{\xi}^{m-1} \mathbf{x}\left(\xi_{0}, \omega\right)= & (\mathrm{Id}-\mathcal{G})^{-1}\left(D_{\xi} J\left(\xi_{0}, \omega\right)\left(\xi-\xi_{0}\right)-R^{m}\right) \\
+ & (\mathrm{Id}-\mathcal{G})^{-1}\left(J(\xi, \omega)-J\left(\xi_{0}, \omega\right)\right. \\
& \left.-D_{\xi} J\left(\xi_{0}, \omega\right)\left(\xi-\xi_{0}\right)+I^{m}\right) .
\end{aligned}
$$

Using the same method as for $I$, by inductive assumption we obtain

$$
\left\|I^{m}\right\|_{L^{m-1}\left(E^{c u}, C_{m \gamma(\omega)+\zeta(\omega)}^{-}\right)}=o\left(\left|\xi-\xi_{0}\right|\right),
$$

as $\xi \rightarrow \xi_{0}$. Furthermore, 


$$
\begin{array}{r}
\left\|J(\xi, \omega)-J\left(\xi_{0}, \omega\right)-D_{\xi} J\left(\xi_{0}, \omega\right)\left(\xi-\xi_{0}\right)+I^{m}\right\|_{L^{m-1}\left(E^{c u}, C_{m \gamma(\omega)+\zeta(\omega)}^{-}\right)} \\
=o\left(\left|\xi-\xi_{0}\right|\right),
\end{array}
$$

as $\xi \rightarrow \xi_{0}$. Hence, $D_{\xi}^{m} \mathbf{x}(\xi, \omega)$ exists and

$$
D_{\xi}^{m} \mathbf{x}(\xi, \omega) \in L^{m}\left(E^{c u}, C_{m \gamma(\omega)+\zeta(\omega)}^{-}\right)
$$

From equation (3.18), we find $D_{\xi}^{m} \mathbf{x}(\cdot, \omega): E^{c u} \rightarrow L^{m}\left(E^{c u}\right.$, $\left.C_{m \gamma(\omega)+\zeta(\omega)}^{-}\right)$satisfies

$$
\begin{aligned}
D_{\xi}^{m} x_{n}(\xi, \omega) & =-\sum_{i=n}^{-1} \Phi^{c u}\left(n-1-i, \theta^{i+1} \omega\right) D_{x x} F_{\rho}^{c u}\left(\theta^{i} \omega, x_{i}(\xi, \omega)\right) \\
\times D_{\xi} x_{i}(\xi, \omega) D_{\xi}^{m-1} x_{i}(\xi, \omega)- & \sum_{i=n}^{-1} \Phi^{c u}\left(n-1-i, \theta^{i+1} \omega\right) D_{x} F_{\rho}^{c u}\left(\theta^{i} \omega, x_{i}(\xi, \omega)\right) \\
\times D_{\xi}^{m} x_{i}(\xi, \omega)+ & \sum_{i=-\infty}^{n-1} \Phi^{s}\left(n-1-i, \theta^{i+1} \omega\right) D_{x x} F_{\rho}^{s}\left(\theta^{i} \omega, x_{i}(\xi, \omega)\right) \\
\times D_{\xi} x_{i}(\xi, \omega) D_{\xi}^{m-1} x_{i}(\xi, \omega)+ & \sum_{i=-\infty}^{n-1} \Phi^{s}\left(n-1-i, \theta^{i+1} \omega\right) D_{x} F_{\rho}^{s}\left(\theta^{i} \omega, x_{i}(\xi, \omega)\right) \\
\times D_{\xi}^{m} x_{i}(\xi, \omega)- & \sum_{i=n}^{-1} \Phi^{c u}\left(n-1-i, \theta^{i+1} \omega\right) D_{\xi} R_{m-1, i}^{c u}(\xi, \omega) \\
& +\sum_{i=-\infty}^{n-1} \Phi^{s}\left(n-1-i, \theta^{i+1} \omega\right) D_{\xi} R_{m-1, i}^{s}(\xi, \omega) \\
\times D_{\xi}^{m} x_{i}(\xi, \omega)+ & \sum_{i=-\infty}^{-1} \Phi^{s}\left(-1-i, \theta^{i+1} \omega\right) D_{\xi} R_{m-1, i}^{s}(\xi, \omega) . \\
\times D_{\xi} x_{i}(\xi, \omega) D_{\xi}^{m-1} x_{i}(\xi, \omega)+ & \sum_{i=-\infty}^{-1} \Phi^{s}\left(-1-i, \theta^{i+1} \omega\right) D_{x} F_{\rho}^{s}\left(\theta^{i} \omega, x_{i}(\xi, \omega)\right) \\
D_{\xi}^{m} x_{0}(\xi, \omega)= & \sum_{\left.i=-1, \theta^{i+1} \omega\right) D_{x x} F_{\rho}^{s}\left(\theta^{i} \omega, x_{i}(\xi, \omega)\right)} \\
&
\end{aligned}
$$


Set

$$
K_{m}(\omega):=\frac{L(\alpha, \beta, m \gamma+\zeta)\left[K^{*}(\omega) K_{1}(\omega) K_{m-1}(\omega)+K_{*}(\omega) \widetilde{K}_{m}(\omega)\right]}{1-(1 / 3) L_{1}(\alpha, \beta)^{-1} L(\alpha, \beta, m \gamma+\zeta)} .
$$

By simple computation, we have

$$
\left\|D_{\xi}^{m} \mathbf{x}(\xi, \omega)\right\|_{L^{m}\left(E^{c u}, C_{m \gamma(\omega)+\zeta(\omega)}^{-}\right)} \leq K_{m}(\omega) .
$$

Next we prove that $D_{\xi}^{m} \mathbf{x}(\cdot, \omega): E^{c u} \rightarrow L^{m}\left(E^{c u}, C_{m \gamma(\omega)}^{-}\right)$is continuous. We change $\xi_{0}$ to $\xi$ on the right hand side of equation (3.10) and define the operator as $\mathcal{G}_{1}: L^{m}\left(E^{c u}, C_{m \gamma(\omega)}^{-}\right) \rightarrow L^{m}\left(E^{c u}, C_{m \gamma(\omega)}^{-}\right)$. Let $Q:=\left\{Q_{n}\right\}_{n \in \mathbb{Z}^{-}}$, where

$$
\begin{gathered}
Q_{n}=-\sum_{i=n}^{-1} \Phi^{c u}\left(n-1-i, \theta^{i+1} \omega\right) D_{x x} F_{\rho}^{c u}\left(\theta^{i} \omega, x_{i}(\xi, \omega)\right) \\
\times D_{\xi} x_{i}(\xi, \omega) D_{\xi}^{m-1} x_{i}(\xi, \omega)+\sum_{i=-\infty}^{n-1} \Phi^{s}\left(n-1-i, \theta^{i+1} \omega\right) D_{x x} F_{\rho}^{s}\left(\theta^{i} \omega, x_{i}(\xi, \omega)\right) \\
\times D_{\xi} x_{i}(\xi, \omega) D_{\xi}^{m-1} x_{i}(\xi, \omega)-\sum_{i=n}^{-1} \Phi^{c u}\left(n-1-i, \theta^{i+1} \omega\right) D_{\xi} R_{m-1, i}^{c u}(\xi, \omega) \\
+\sum_{i=-\infty}^{n-1} \Phi^{s}\left(n-1-i, \theta^{i+1} \omega\right) D_{\xi} R_{m-1, i}^{s}(\xi, \omega) \\
Q_{0}=\sum_{i=-\infty}^{-1} \Phi^{s}\left(-1-i, \theta^{i+1} \omega\right) D_{x x} F_{\rho}^{s}\left(\theta^{i} \omega, x_{i}(\xi, \omega)\right) \\
\times D_{\xi} x_{i}(\xi, \omega) D_{\xi}^{m-1} x_{i}(\xi, \omega)+\sum_{i=-\infty}^{-1} \Phi^{s}\left(-1-i, \theta^{i+1} \omega\right) D_{\xi} R_{m-1, i}^{s}(\xi, \omega) .
\end{gathered}
$$

From equations (3.23) and (3.10) we have

$$
D_{\xi}^{m} \mathbf{x}(\xi, \omega)-D_{\xi}^{m} \mathbf{x}\left(\xi_{0}, \omega\right)=\mathcal{G}_{1}\left(D_{\xi}^{m} \mathbf{x}(\xi, \omega)-D_{\xi}^{m} \mathbf{x}\left(\xi_{0}, \omega\right)\right)+Q .
$$

Obviously, $\left\|\mathcal{G}_{1}\right\| \leq 1 / 3$. This implies that $\mathrm{Id}-\mathcal{G}_{1}$ has a bounded inverse in $L^{m}\left(E^{c u}, C_{m \gamma(\omega)}^{-}\right)$. By the same procedure as for $H$, we can prove 
that

$$
\|Q\|_{L^{m}\left(E^{c u}, C_{m \gamma(\omega)}^{-}\right)}=o(1) \quad \text { as } \xi \rightarrow \xi_{0} .
$$

Then $D_{\xi}^{m} \mathbf{x}(\xi, \omega)$ is continuous in $L^{m}\left(E^{c u}, C_{m \gamma(\omega)}^{-}\right)$. By inductive assumption, the results in Lemma 3.2 (iii)-(iv) are established.

It remains to prove that $\mathbf{x}(\cdot, \omega) \in C^{N, \tau(\omega)}$. Clearly, for each

$$
\gamma \in\left[-\frac{\beta(\omega)}{2(N+1)},-\frac{\beta(\omega)}{2(N+1)^{2}}\right],
$$

$\mathbf{x}(\cdot, \omega)$ is $C^{i}$ from $E^{c u}$ to $C_{(i+\tau(\omega)) \gamma(\omega)}^{-}$for $i=1, \ldots, N$. Following equations (3.9), (3.15) and (3.17), Lemmas 2.13 and 2.16,

$$
\begin{aligned}
& \left\|D_{\xi} x_{i}(\xi, \omega)-D_{\xi} x_{i}\left(\xi_{0}, \omega\right)\right\| e^{-(1+\tau(\omega)) \gamma(\omega) i} \\
\leq \frac{1}{3} L_{1}(\alpha, \beta)^{-1} L(\alpha, \beta,(1+\tau) \gamma) \| D_{\xi} \mathbf{x}(\xi, \omega)- & D_{\xi} \mathbf{x}\left(\xi_{0}, \omega\right) \|_{(1+\tau(\omega)) \gamma(\omega)}^{-} \\
& +V_{1}^{a}(\omega)\left|\xi-\xi_{0}\right|^{\tau(\omega)}
\end{aligned}
$$

where

$$
V_{1}^{a}(\omega):=\left(\frac{3 K(\omega)}{2}\right)^{\tau(\omega)} \underline{A}_{1}(\omega) K_{1}(\omega) L(\alpha, \beta,(1+\tau) \gamma)
$$

and $\underline{A}_{1}(\omega)$ is the tempered-from-above random variable such that $K\left(\theta^{i+1} \omega\right) A_{1}\left(\theta^{i} \omega\right) e^{\zeta(\omega) i} \leq \underline{A}_{1}(\omega)$. Then, by equation (3.8), we get that

$$
\begin{aligned}
\| D_{\xi} \mathbf{x}(\xi, \omega)- & D_{\xi} \mathbf{x}\left(\xi_{0}, \omega\right) \|_{(1+\tau(\omega)) \gamma(\omega)}^{-} \\
& \leq \frac{V_{1}^{a}(\omega)}{1-(1 / 3) L_{1}(\alpha, \beta)^{-1} L(\alpha, \beta,(1+\tau) \gamma)}\left|\xi-\xi_{0}\right|^{\tau(\omega)} .
\end{aligned}
$$

Let

$$
K_{1}^{a}(\omega):=\frac{V_{1}^{a}(\omega)}{1-(1 / 3) L_{1}(\alpha, \beta)^{-1} L(\alpha, \beta,(1+\tau) \gamma)} .
$$

We assume that, for all $1 \leq j \leq m-1,2 \leq m \leq N$, there exist random variables tempered from above $K_{j}^{a}(\omega)$ such that

$$
\left\|D_{\xi}^{j} \mathbf{x}(\xi, \omega)-D_{\xi}^{j} \mathbf{x}\left(\xi_{0}, \omega\right)\right\|_{(j+\tau(\omega)) \gamma(\omega)}^{-} \leq K_{j}^{a}(\omega)\left|\xi-\xi_{0}\right|^{\tau(\omega)}
$$

and prove it for $j=m$. By inductive assumption, we obtain that, for each $\xi, \xi_{0} \in E^{c u}$ and $\tau=s, u$, 


$$
\begin{aligned}
& \left\|D_{\xi} R_{m-1, i}^{\tau}(\xi, \omega)-D_{\xi} R_{m-1, i}^{\tau}\left(\xi_{0}, \omega\right)\right\| \\
& \leq\left[\bar{A}_{m}\left(\theta^{i} \omega\right) \widetilde{K}_{m}(\omega) e^{[\gamma(\omega)(m+\tau(\omega))+2 \zeta(\omega)] i}\left(\frac{3 K(\omega)}{2}\right)^{\tau(\omega)}\right. \\
& \left.\quad+\bar{B}_{m}\left(\theta^{i} \omega\right) \widetilde{\widetilde{K}}_{m}(\omega) e^{[\gamma(\omega)(m+\tau(\omega))+2 \zeta(\omega)] i}\right]\left|\xi-\xi_{0}\right|^{\tau(\omega)},
\end{aligned}
$$

where $\bar{A}_{m}(\omega)=\max _{0 \leq i \leq m} A_{i}(\omega), \bar{B}_{m}(\omega)=\max _{1 \leq i \leq m} B_{i}(\omega)$ and $\widetilde{K}_{m}(\omega)$ and $\widetilde{\widetilde{K}}_{m}(\omega)$ are, respectively, the $m$ th-order polynomial of $K_{1}(\omega), \ldots, K_{m-1}(\omega)$ and the $m$ th-order polynomial of $K_{1}(\omega), K_{1}^{a}(\omega)$, $\ldots, K_{m-1}(\omega), K_{m-1}^{a}(\omega)$ with positive integer coefficients that are tempered from above.

Applying Lemma 2.13 again, we directly see that $K\left(\theta^{i+1} \omega\right) \times$ $A_{2}\left(\theta^{i} \omega\right) e^{2 \zeta(\omega) i}, K\left(\theta^{i+1} \omega\right) \bar{A}_{m}\left(\theta^{i} \omega\right) e^{2 \zeta(\omega) i}, K\left(\theta^{i+1} \omega\right) B_{2}\left(\theta^{i} \omega\right) e^{\zeta(\omega) i}$ and $K\left(\theta^{i+1} \omega\right) \bar{B}_{m}\left(\theta^{i} \omega\right) e^{2 \zeta(\omega) i}$ are bounded by tempered-from-above random variables $\underline{A}_{2}(\omega), \underline{A}_{m}(\omega), \underline{B}_{2}(\omega)$ and $\underline{B}_{m}(\omega)$ independent of $i$. Using equations (3.23) and (3.24), by simple calculations we have

$$
\begin{aligned}
& \left\|D_{\xi}^{m} x_{i}(\xi, \omega)-D_{\xi}^{m} x_{i}\left(\xi_{0}, \omega\right)\right\| e^{-(m+\tau(\omega)) \gamma(\omega) i} \\
& \quad \leq \frac{1}{3} L_{1}(\alpha, \beta)^{-1} L(\alpha, \beta,(m+\tau) \gamma) \\
& \times\left\|D_{\xi}^{m} \mathbf{x}(\xi, \omega)-D_{\xi}^{m} \mathbf{x}\left(\xi_{0}, \omega\right)\right\|_{(m+\tau(\omega)) \gamma(\omega)}^{-}+K^{a}(\omega)\left|\xi-\xi_{0}\right|^{\tau(\omega)},
\end{aligned}
$$

where

$$
\begin{aligned}
& K^{a}(\omega):= {\left[\underline{A}_{2}(\omega)\left(\frac{3 K(\omega)}{2}\right)^{\tau(\omega)} K_{1}(\omega) K_{m-1}(\omega)\right.} \\
&+\underline{B}_{2}(\omega) K_{1}^{a}(\omega) K_{m-1}(\omega)+\underline{B}_{2}(\omega) K_{1}(\omega) K_{m-1}^{a}(\omega) \\
&+\underline{A}_{1}(\omega)\left(\frac{3 K(\omega)}{2}\right)^{\tau(\omega)} K_{m}(\omega) \\
&\left.+\underline{A}_{m}(\omega) \widetilde{K}_{m}(\omega)\left(\frac{3 K(\omega)}{2}\right)^{\tau(\omega)}+\underline{B}_{m}(\omega) \widetilde{\widetilde{K}}_{m}(\omega)\right] \\
& L(\alpha, \beta,(m+\tau) \gamma) .
\end{aligned}
$$

Set

$$
K_{m}^{a}(\omega):=\frac{K^{a}(\omega)}{1-(1 / 3) L_{1}(\alpha, \beta)^{-1} L(\alpha, \beta,(m+\tau) \gamma)} .
$$


Then we obtain that

$$
\left\|D_{\xi}^{m} \mathbf{x}(\xi, \omega)-D_{\xi}^{m} \mathbf{x}\left(\xi_{0}, \omega\right)\right\|_{(m+\tau(\omega)) \gamma(\omega)}^{-} \leq K_{m}^{a}(\omega)\left|\xi-\xi_{0}\right|^{\tau(\omega)} .
$$

It follows from inductive assumption that the proof of Lemma 3.2 is complete.

Now we provide the center-unstable manifold theorem.

Theorem 3.3 (Center-unstable manifold theorem). Assume that Hypotheses 2.7-2.14 hold. Then the center-unstable set is a $C^{N}$ manifold, which is given by

$$
W^{c u}(\omega)=\left\{\xi+h^{c u}(\xi, \omega) \mid \xi \in E^{c u}\right\},
$$

where $h^{c u}: E^{c u} \times \Omega \rightarrow E^{s}$ satisfies the following:

(i) $h^{c u}(\xi, \omega)$ is $\mathcal{B}\left(E^{c u}\right) \otimes \mathcal{F}$-measurable and $h^{c u}(\xi, \omega)$ is $C^{N}$ in $\xi$ with

$$
\operatorname{Lip} h^{c u}(\cdot, \omega)<1, \quad h^{c u}(0, \omega)=0, \quad D_{\xi} h^{c u}(0, \omega)=0 ;
$$

(ii) $\left\|D_{\xi}^{i} h^{c u}(\xi, \omega)\right\| \leq \widehat{K}_{i}(\omega)$ for all $0 \leq i \leq N<+\infty$, where each $\widehat{K}_{i}(\omega)$ is random variable tempered from above;

(iii) $W_{\text {loc }}^{c u}(\omega)=\left\{x \in W^{c u}(\omega) \mid x \in B(0, \rho(\omega))\right\}$ is a local unstablecenter manifold of the original system (2.1);

(iv) in addition, if Hypothesis 2.15 holds, $h^{c u}(\cdot, \omega) \in C^{N, \tau(\omega)}$, and there is a random variable tempered from above $\widehat{K}_{N+1}(\omega)$ such that

$$
\left\|D_{\xi}^{N} h^{c u}(\xi, \omega)-D_{\xi}^{N} h^{c u}\left(\xi_{0}, \omega\right)\right\| \leq \widehat{K}_{N+1}(\omega)\left|\xi-\xi_{0}\right|^{\tau(\omega)} \text { for all } \xi, \xi_{0} \in E^{c u}
$$

Proof. We define $h^{c u}: E^{c u} \times \Omega \rightarrow E^{s}$ by

$$
\begin{aligned}
h^{c u}(\xi, \omega) & :=x_{0}^{s}(\xi, \omega) \\
& =\sum_{i=-\infty}^{-1} \Phi^{s}\left(-1-i, \theta^{i+1} \omega\right) F_{\rho}^{s}\left(\theta^{i} \omega, x_{i}(\xi, \omega)\right) .
\end{aligned}
$$

By Lemma 3.1, we see that

$$
W^{c u}(\omega)=\left\{x_{0}(\xi, \omega) \mid \xi \in E^{c u}\right\}=\left\{\xi+h^{c u}(\xi, \omega) \mid \xi \in E^{c u}\right\} .
$$


Take

$$
\gamma(\omega):=-\frac{\beta(\omega)}{2(N+1)^{2}},
$$

and let $\Theta(\omega):=-\gamma(\omega)$ in equation (2.4). For every $\xi, \xi_{0} \in E^{c u}$, we have that

$$
\begin{aligned}
& \left|h^{c u}(\xi, \omega)-h^{c u}\left(\xi_{0}, \omega\right)\right| \\
& \leq \sum_{i=-\infty}^{-1} 10 K\left(\theta^{i+1} \omega\right) \widetilde{B}_{2}\left(\theta^{i} \omega\right) \rho\left(\theta^{i} \omega\right) e^{-\beta(\omega)(-1-i)} e^{\gamma(\omega) i} \\
& \times\left|\mathbf{x}(\xi, \omega)-\mathbf{x}\left(\xi_{0}, \omega\right)\right|_{\gamma(\omega)}^{-} .
\end{aligned}
$$

Since

$$
\left|\mathbf{x}(\xi, \omega)-\mathbf{x}\left(\xi_{0}, \omega\right)\right|_{\gamma(\omega)}^{-} \leq \frac{3 K(\omega)}{2}\left|\xi-\xi_{0}\right|,
$$

together with $K(\omega) \leq K\left(\theta^{i} \omega\right) e^{-\gamma(\omega)|i|}$, we have

$$
\left|h^{c u}(\xi, \omega)-h^{c u}\left(\xi_{0}, \omega\right)\right| \leq \frac{e^{\beta(\omega)} L_{1}(\alpha, \beta)^{-1}}{2}\left|\xi-\xi_{0}\right| \sum_{i=-\infty}^{-1} e^{(2 \gamma(\omega)+\beta(\omega)) i} .
$$

Thus, we obtain

$$
\left|h^{c u}(\xi, \omega)-h^{c u}\left(\xi_{0}, \omega\right)\right| \leq \frac{1}{2} L_{1}(\alpha, \beta)^{-1} L(\alpha, \beta, 2 \gamma)\left|\xi-\xi_{0}\right| \leq \frac{1}{2}\left|\xi-\xi_{0}\right| .
$$

It is clear that (i)-(ii) follow from Lemma 3.2 (ii)-(iv), and (iii) is true. Moreover, measurability of $W^{c u}(\omega)$ is a consequence of the continuity and measurability of $h^{c u}(\xi, \omega)$.

To this end, we note inequality (3.25) and obtain

$$
\begin{aligned}
\left\|D_{\xi}^{N} h^{c u}(\xi, \omega)-D_{\xi}^{N} h^{c u}\left(\xi_{0}, \omega\right)\right\| & =\left\|P^{s}\left(D_{\xi}^{N} x_{0}(\xi, \omega)-D_{\xi}^{N} x_{0}\left(\xi_{0}, \omega\right)\right)\right\| \\
& \leq K_{N}^{a}(\omega)\left|\xi-\xi_{0}\right|^{\tau(\omega)} .
\end{aligned}
$$

Let $\widehat{K}_{N+1}(\omega):=K_{N}^{a}(\omega)$. The proof is complete.

The existence and Hölder continuity of smooth center-stable manifolds of the random dynamical system $\varphi(n, \omega, x)$ are established in exactly the same way. We summarize the center-stable manifold theorem as follows. 
Theorem 3.4 (Center-stable manifold theorem). Assume that $\mathrm{Hy}$ potheses 2.7-2.14 hold. Then the center-stable set is a $C^{N}$ manifold which is given by

$$
W^{c s}(\omega)=\left\{\xi+h^{c s}(\xi, \omega) \mid \xi \in E^{c s}\right\},
$$

where $h^{c s}: E^{c s} \times \Omega \rightarrow E^{u}$ satisfies the following:

(i) $h^{c s}(\xi, \omega)$ is $\mathcal{B}\left(E^{c s}\right) \otimes \mathcal{F}$-measurable and $h^{c s}(\xi, \omega)$ is $C^{N}$ in $\xi$ with

$$
\operatorname{Lip} h^{c s}(\cdot, \omega)<1, h^{c s}(0, \omega)=0, D_{\xi} h^{c s}(0, \omega)=0 ;
$$

(ii) for all $0 \leq i \leq N<+\infty$, we have that $\left\|D_{\xi}^{i} h^{c s}(\xi, \omega)\right\| \leq \bar{K}_{i}(\omega)$, where each $\bar{K}_{i}(\omega)$ is random variable tempered from above;

(iii) $W_{\text {loc }}^{c s}(\omega)=\left\{x \in W^{c s}(\omega) \mid x \in B(0, \rho(\omega))\right\}$ is a local center-stable manifold of the original system (2.1);

(iv) in addition, if Hypothesis 2.15 holds, $h^{c s}(\cdot, \omega) \in C^{N, \tau(\omega)}$ and there is a random variable tempered from above $\bar{K}_{N+1}(\omega)$ such that $\left\|D_{\xi}^{N} h^{c s}(\xi, \omega)-D_{\xi}^{N} h^{c s}\left(\xi_{0}, \omega\right)\right\| \leq \bar{K}_{N+1}(\omega)\left|\xi-\xi_{0}\right|^{\tau(\omega)}$ for all $\xi, \xi_{0} \in E^{c s}$.

Since the above preparations have been finished, we are now ready to prove the center manifold theorem.

Theorem 3.5 (Center manifold theorem). Assume that Hypotheses 2.7-2.14 hold. Then the center set is a $C^{N}$ manifold, which is given by

$$
W^{c}(\omega)=\left\{\zeta+h^{c}(\zeta, \omega) \mid \zeta \in E^{c}\right\},
$$

where $h^{c}: E^{c} \times \Omega \rightarrow E^{u} \oplus E^{s}$ satisfies the following:

(i) $h^{c}(\zeta, \omega)$ is $\mathcal{B}\left(E^{c}\right) \otimes \mathcal{F}$-measurable and Lipschitz continuous with respect to $\zeta$

(ii) $h^{c}(\zeta, \omega)$ is $C^{N}$ in $\zeta$ with $h^{c}(0, \omega)=0$ and $D_{\zeta} h^{c}(0, \omega)=0$, and for each $0 \leq i \leq N<+\infty$, there exists a random variable tempered from above $K_{i}^{\prime}(\omega)$ such that $\left\|D_{\zeta}^{i} h^{c}(\zeta, \omega)\right\| \leq K_{i}^{\prime}(\omega)$;

(iii) $W_{\mathrm{loc}}^{c}(\omega)=\left\{x \in W^{c}(\omega) \mid x \in B(0, \rho(\omega))\right\}$ is a local center manifold of the original system (2.1);

(iv) in addition, if Hypothesis 2.15 holds, then $h^{c}(\cdot, \omega) \in C^{N, \tau(\omega)}$, and there is a random variable tempered from above $K_{N+1}^{\prime}(\omega)$ such that

$$
\left\|D_{\zeta}^{N} h^{c}(\zeta, \omega)-D_{\zeta}^{N} h^{c}\left(\zeta_{0}, \omega\right)\right\| \leq K_{N+1}^{\prime}(\omega)\left|\zeta-\zeta_{0}\right|^{\tau(\omega)} \text { for all } \zeta, \zeta_{0} \in E^{c} .
$$


Proof. Obviously, (iii) is true. For any $x \in W^{c}(\omega)$, we have $x=\xi+\eta+\zeta$, where $\xi \in E^{u}, \eta \in E^{s}$ and $\zeta \in E^{c}$. Note that $x \in W^{c u}$ and $x \in W^{c s}$. By Theorem 3.3, we have $\eta=h^{c u}(\xi+\zeta, \omega)$. Similarly, Theorem 3.4 implies $\xi=h^{c s}(\eta+\zeta, \omega)$. Thus,

$$
\eta=h^{c u}\left(h^{c s}(\eta+\zeta, \omega)+\zeta, \omega\right) .
$$

For each fixed $\omega \in \Omega$, by Theorems 3.3 (i) and 3.4 (i), $\operatorname{Lip} h^{c u}(\cdot, \omega)$. $\operatorname{Lip} h^{c s}(\cdot, \omega)<1$. Moreover, recall that $h^{c s}(\cdot, \omega), h^{c u}(\cdot, \omega) \in C^{N}$. It then follows from the contraction mapping principle with a parameter that there exists a $C^{N}$ mapping $h_{1}^{c}: E^{c} \times \Omega \rightarrow E^{s}$ such that $\eta=h_{1}^{c}(\zeta, \omega)$. Similarly, there exists a $C^{N}$ mapping $h_{2}^{c}: E^{c} \times \Omega \rightarrow E^{u}$ such that $\xi=h_{2}^{c}(\zeta, \omega)$. Instead of $h^{c}(\zeta, \omega)$, it is not difficult to see that both $h_{1}^{c}(\zeta, \omega)$ and $h_{2}^{c}(\zeta, \omega)$ satisfy $(\mathrm{i})$, and by the inductive approach that they satisfy (ii) and (iv). Set

$$
h^{c}(\zeta, \omega):=h_{1}^{c}(\zeta, \omega)+h_{2}^{c}(\zeta, \omega) .
$$

The proof is complete.

\section{REFERENCES}

1. L. Arnold, Random dynamical systems, Springer, New York, 1998.

2. P. Bates and C. Jones, Invariant maniflods for semilinear partial differential equations, Dyn. Rep. 2 (1989), 1-38.

3. P. Bates, K. Lu and C. Zeng, Existence and persistence of invariant manifolds for semiflows in Banach space, Mem. Amer. Math. Soc. 135, American Mathematical Society, Providence, 1998.

4. __ Invariant foliations near normally hyperbolic invariant manifolds for semiflows, Trans. Amer. Math. Soc. 352 (2000), 4641-4676.

5. , Approximately invariant manifolds and global dynamics of spike states, Invent. Math. 174 (2008), 355-433.

6. J. Carr, Application of centre manifold theory, Springer-Verlag, New York, 1981.

7. C. Castaing and M. Valadier, Convex analysis and measurable multifunctions, Lect. Notes Math. 580, Springer, Berlin, 1977.

8. S.-N. Chow, C. Li and D. Wang, Normal forms and bifurcation of planar vector fields, Cambridge University Press, London, 1994.

9. S-N. Chow, X. Lin and K. Lu, Smooth invariant foliations in infinite dimensional spaces, J. Diff. Eqs. 94 (1991), 266-291.

10. S.-N. Chow, W. Liu and Y. Yi, Center manifolds for invariant sets, J. Diff. Eqs. 168 (2000), 355-385. 
11. S.-N. Chow, W. Liu and Y. Yi, Center manifolds for smooth invariant manifolds, Trans. Amer. Math. Soc. 352 (2000), 5179-5211.

12. S-N. Chow and K. Lu, Invariant manifolds for flows in Banach spaces, J. Diff. Eqs. 74 (1988), 285-317.

13.,$C^{k}$ center unstable manifolds, Proc. Roy. Soc. Edinb. 108 (1988), 303-320.

14. B. Duan, K. Lu and B. Schmalfuß, Invariant manifolds for stochastic partial differential equations, Ann. Prob. 31 (2003), 2109-2135.

15. Smooth stable and unstable manifolds for stochastic evolutionary equations, J. Dyn. Diff. Eqs. 16 (2004), 949-972.

16. J. Hadamard, Surl'iteration et les solutions asymptotiquesd es equations differentielles, Bull. Soc. Math. France 29 (1901), 224-228.

17. J.K. Hale, Ordinary differential equations, John Wiley, New York, 1969.

18. D. Henry, Geometric theory of semilinear parabolic equations, Lect. Notes Math. 840, Springer-Verlag, New York, 1981.

19. A. Kelley, The stable, center-stable, center, center-unstable, unstable manifolds, J. Diff. Eqs. 3 (1967), 546-570.

20. W. Li and K. Lu, Sternberg theorems for random dynamical systems, Comm. Pure Appl. Math. 58 (2005), 941-988.

21. Z. Lian and K. Lu, Lyapunov exponents and invariant manifolds for random dynamical systems in a Banach space, Mem. Amer. Math. Soc. 206 (2010), 1-106.

22. K. Lu and B. Schmalfuß, Invariant manifolds for stochastic wave equations, J. Diff. Eqs. 236 (2007), 460-492.

23. Invariant foliations for stochastic partial differential equations, Stoch. Dyn. 8 (2008), 505-518.

24. A.M. Lyapunov, Problème géneral de la stabilité du mouvement, Princeton University Press, Princeton, 1947.

25. S.-E.A. Mohammed and M.K.R. Scheutzow, The stable manifold theorem for stochastic differential equations, Ann. Prob. 27 (1999), 615-652.

26. O. Perron, Über Stabilität und asymptotisches Verhalten der Integrale von Differentialgleichungssysteme, Math. Z. 29 (1928), 129-160.

27. V.A. Pliss, Principal reduction in the theory of stability of motion, Izv. Akad. Nauk 28 (1964), 1297-1324 (in Russian).

28. B. Schmalfuß, A random fixed point theorem and the random graph transformation, J. Math. Anal. Appl. 225 (1998), 91-113.

29. A. Vanderbauwhede and S.A. van Gils, Center manifolds and contractions on a scale of Bannach spaces, J. Funct. Anal. 72 (1987), 209-224. 
30. T. Wanner, Linearization of random dynamical systems, Dyn. Rep. 4 (1995), 203-268.

School of Mathematics, Sichuan University, Chengdu, Sichuan 610064, P.R. CHINA

Email address: guopengcn@126.com

Academy of Mathematics and System Sciences Chinese Academy of Sciences, Haidian District, Beijing 100190, P.R. China

Email address: junshen85@163.com 